\title{
SINGULAR LOCI OF COMINUSCULE SCHUBERT VARIETIES
}

\author{
C. ROBLES
}

\begin{abstract}
Let $X=G / P$ be a cominuscule rational homogeneous variety. Equivalently, $X$ admits the structure of a compact Hermitian symmetric space. I give a uniform description (that is, independent of type) of the irreducible components of the singular locus of a Schubert variety $Y \subset X$ in terms of representation theoretic data. The result is based on a recent characterization of the Schubert varieties by an integer $a \geq 0$ and a marked Dynkin diagram. Corollaries include: (1) the variety is smooth if and only if $a=0 ;(2)$ if $G$ of Type ADE, then the singular locus occurs in codimension at least three.
\end{abstract}

\section{INTRODUCTION}

Let $X=G / P$ be a cominuscule rational homogeneous variety. (Equivalently, $X$ admits the structure of a compact Hermitian symmetric space. An example is the Grassmannian $\operatorname{Gr}(k, n)$ of $k$-planes in complex $n$-space.) The main result (Theorem 3.3) of this paper is a uniform (independent of $G$ ) description of the irreducible components of the singular loci of Schubert varieties $Y \subset X$.

Context and related results. It is an important open question, to explicitly describe the singular loci of Schubert varieties in an arbitrary (not necessarily cominuscule) rational homogeneous variety $X=G / P$. The problem lies at the interface between geometry, combinatorics and representation theory, and has stimulated research in each area. There is a vast body of literature on the subject, and the interested reader may consult [1] for an excellent overview.

In general, characterizations of smooth Schubert varieties, and descriptions of singular loci tend to be combinatorial in nature. As an example, consider the full flag variety $X=\mathrm{SL}_{n} \mathbb{C} / B$, where $B$ is a Borel subgroup (e.g., upper triangular matrices). In this case, the Schubert varieties are indexed by the symmetric group $S_{n}$ on $n$ letters. Given $w \in S_{n}$, V. Lakshmibai and B. Sandhya [10] showed that the corresponding Schubert variety $X_{w}$ is smooth if and only if the permutation avoids the patterns (3412) and (4231). At that time, they gave a conjectural (and combinatorial) description of the irreducible components (which are necessarily Schubert varieties) of singular locus. Gasharov [7] established the sufficiency of the Lakshmibai-Sandhya conditions, and the full conjecture was established (independently) by S. Billey and G. Warrington [3], A. Cortez [6], C. Kassel, A. Lascoux and C. Reutenaur [8], and L. Manivel [12]. More recently, Billey and Postnikov [2] have

Date: November 19, 2018.

2010 Mathematics Subject Classification. 14M15.

Key words and phrases. Rational homogeneous varieties, Schubert variety, cominuscule, compact Hermitian symmetric space, singular locus.

Robles is partially supported by NSF DMS-1006353. 
uniformly extended the Lakshmibai-Sandhya smoothness criterion to generalized flag manifolds $X=G / B$. An advantage of the pattern avoidance criteria over other characterizations (see [1]) of smoothness (and the weaker rational smoothness) is that it is nonrecursive and computationally efficient.

Returning to the case that $X=G / P$ is cominuscule (in general, $P$ is not a Borel subgroup), type-specific descriptions in the case that $X$ is classical ( $G$ is of type ABCD) have been known for some time: the type A case ( $X$ is a Grassmannian) since the 1970s, and the rest by 1990; see [1, Section 9.3] and the references therein. Those descriptions are given in terms of partitions. In contrast, the characterization of Theorem 3.3 is given by representation theoretic data, and so presents a complimentary perspective. It also has the advantage of being independent of type, and yields an explicit description of the singular loci in the two non-classical cases $\left(G=E_{6}, E_{7}\right)$. Like the pattern advoidence criteria discussed above, the characterization is both nonrecursive and amenable to compuation.

The cominuscule $X$ are closely related to the minuscule rational homogeneous varieties. Indeed, with the exception of the quadric hypersurface $\mathcal{Q}^{2 n-1}=B_{n} / P_{1}$ and the Lagrangian Grassmannian LG $(n, 2 n)=C_{n} / P_{n}$, every irreducible cominuscule $X$ is minuscule. Theorem 3.3 compliments descriptions of the singular loci of Schubert varieties in minuscule $X$ by M. Brion and P. Polo [4], and by N. Perrin [13]. (Brion and Polo also study singularities of cominuscule Schubert varieties, but - so far as I can discern - stop short of a complete description of the irreducible components.)

Contents. In Section 2 we review Schubert varieties, and their representation theoretic characterization by an integer $\mathrm{a} \geq 0$ and a marking $\mathrm{J}$ of the Dynkin diagram of $G$. (The pair (a, J) encodes the relationship between $P$ and the stabilizer of the Schubert variety; see Remark 4.7.) The main theorem and subsequent corollaries are discussed in Section 3. Corollaries 3.5 and 3.6 describe the relationship between the integer $\mathrm{a}=\mathrm{a}(Y)$ and the number of irreducible components in $\operatorname{Sing}(Y)$. For example, $Y$ is smooth if and only if $\mathrm{a}=0$; if $\mathrm{a}=1$, then $\operatorname{Sing}(Y)$ is irreducible. Corollary 3.7 gives lower bounds on the codimension of the singular locus, and characterizes those Schubert varieties for which the bound is realized. The main result is proved in Section 4 .

\section{CONTEnts}

1. Introduction 1

2. Review 3

3. Singular locus 5

Table 1: Singular loci of Schubert varieties in $E_{6} / P_{6} \quad 8$

Table 2: Singular loci of Schubert varieties in $E_{7} / P_{7} \quad 9$

4. Proof of Theorem 3.3 10

Appendix A. Geometric descriptions of $Y_{\mathrm{a}, \mathrm{J}} \quad 15$

$\begin{array}{lll}\text { Appendix B. The exceptional cases } & 18\end{array}$

References $\quad 18$

Figure 1: Hasse poset and a, J data for the Cayley plane $E_{6} / P_{6} \quad 21$

Figure 2: Hasse poset and a, J data for the Freudenthal variety $E_{7} / E_{7} \quad 22$ 
1.1. Use of LiE. There are two exceptional, irreducible compact Hermitian symmetric spaces: the Cayley plane $E_{6} / P_{6}$ and the Freudenthal variety $E_{7} / P_{7}$. The software [11] is used to perform computations and verify some results for these two cominuscule varieties (Section 3.1 and Appendix B). I emphasize that none of the proofs of the paper rely on LiE; the software is used only to compute the $(\mathrm{a}, \mathrm{J})$-values for these two exceptional varieties. Moreover, the code I wrote applies to any cominuscule variety; that is, it is not specialized to the exceptional cases. This allowed me to test the code by applying it to classical cominuscule varieties (of low rank - the code requires that the rank of $G$ be specified). The outputs are consistent with the "by hand" results obtained for the classical cases. This provides some confidence for the accuracy of the code and the corresponding computations in the two exceptional cases.

Acknowledgements. Over the course of this project, I have benefitted from conversations and/or correspondence with many people, including S. Billey, J. Carrell, V. Lakshmibai, F. Sottile, D. The, A. Woo and A. Yong. I thank them for their insights and time.

\section{REVIEW}

2.1. Notation and background. The present article is founded on a result of [15]. With the exception noted in Remark 2.10, I will use the notation of that paper. To streamline the presentation, I will give a laconic review of the discussion of rational homogeneous varieties, their Schubert subvarieties, grading elements and Hasse diagrams in [15, Sections 2.1-2.4 and 3.1]. Briefly, $G$ is a complex simple Lie group. A choice of Cartan and Borel subgroups $H \subset B$ has been fixed, $P \supset B$ is a maximal parabolic subgroup associated with a cominuscule root, and $X=G / P$ is the corresponding cominuscule variety. The associated Lie algebras are denoted $\mathfrak{h} \subset \mathfrak{b} \subset \mathfrak{p} \subset \mathfrak{g}$. Let $W$ denote the Weyl group of $\mathfrak{g}$, and $W_{\mathfrak{p}}$ the Weyl group of the reductive component in the Levi decomposition of $\mathfrak{p}$. The Hasse diagram $W^{\mathfrak{p}}$ is the set of minimal length representatives of the right-coset space $W_{\mathfrak{p}} \backslash W$, and indexes the Schubert classes. Let

$$
o=P / P \in X=G / P .
$$

Given $w \in W^{\mathfrak{p}}$, the Zariski closure

$$
X_{w}:=\overline{B w^{-1} \cdot o}
$$

is a Schubert variety. Any $G$-translate of the Schubert variety $X_{w}$ will be referred to as a Schubert variety of type $w$. Let $\xi_{w}=\left[X_{w}\right] \in H_{2|w|}(X, \mathbb{Z})$ denote the corresponding Schubert class.

Let $\left\{Z_{1}, \ldots, Z_{r}\right\}$ be the basis of $\mathfrak{h}$ dual to the simple roots $\Sigma=\left\{\alpha_{1}, \ldots, \alpha_{r}\right\}$. Let $\alpha_{\mathfrak{i}}$ be the simple root associated with the cominuscule $\mathfrak{p}$. Since $Z_{i}$ is an element of the Cartan subalgebra $\mathfrak{h}$, the vector space $\mathfrak{g}$ decomposes into a direct sum of $Z_{\mathfrak{i}}$-eigenspaces. The homogeneous variety $G / P$ is cominuscule if and only if the eigenvalues are $\{-1,0,1\}$; that is,

$$
\mathfrak{g}=\mathfrak{g}_{1} \oplus \mathfrak{g}_{0} \oplus \mathfrak{g}_{-1}, \quad \text { where } \quad \mathfrak{g}_{k}:=\left\{A \in \mathfrak{g} \mid\left[Z_{\mathfrak{i}}, A\right]=k A\right\} .
$$

The decomposition (2.1) is the $Z_{\mathfrak{i}}$-graded decomposition of the Lie algebra $\mathfrak{g}$. Moreover,

$$
\mathfrak{p}=\mathfrak{g}_{1} \oplus \mathfrak{g}_{0},
$$

and $\mathfrak{g}_{0}$ is the reductive component of the parabolic subalgebra $\mathfrak{p}$. 
Remark 2.3. As a graded decomposition, we have $\left[\mathfrak{g}_{k}, \mathfrak{g}_{\ell}\right] \subset \mathfrak{g}_{k+\ell}$. In particular, the subspaces $\mathfrak{g}_{ \pm 1}$ are both $\mathfrak{g}_{0}$-modules, and abelian subalgebras of $\mathfrak{g}$.

Equation (2.2) implies

$$
T_{o} X \simeq \mathfrak{g}_{-1}
$$

as an $\mathfrak{g}_{0}$-module.

Notation. Let $\Delta$ denote the set of roots of $\mathfrak{g}$. Given $\alpha \in \Delta$, let $\mathfrak{g}_{\alpha} \subset \mathfrak{g}$ denote the corresponding root space. Given any subset $\mathfrak{s} \subset \mathfrak{g}$, let

$$
\Delta(\mathfrak{s})=\left\{\alpha \in \Delta \mid \mathfrak{g}_{\alpha} \subset \mathfrak{s}\right\} .
$$

Given a subset $U$ of a vector space, let $\langle U\rangle$ denote the linear span.

2.2. The characterization of Schubert varieties. This section is a concise review of the characterization of Schubert varieties $\xi_{w}$ by an integer $\mathrm{a}(w) \geq 0$ and a marking $\mathrm{J}(w)$ of the Dynkin diagram. (The marking is equivalent to a choice of simple roots from $\Sigma \backslash\left\{\alpha_{i}\right\}$.) For more detail see [15]. Given $w \in W^{\mathfrak{p}}$, define

$$
\Delta(w)=w \Delta^{-} \cap \Delta^{+} \subset \Delta\left(\mathfrak{g}_{1}\right) \text { and } \quad \mathfrak{n}_{w}=\bigoplus_{\alpha \in \Delta(w)} \mathfrak{g}_{-\alpha} \subset \mathfrak{g}_{-1} .
$$

The set $\Delta(w)$ is known as the inversion set of $w$. Let $N_{w}=\exp \left(\mathfrak{n}_{w}\right)$. Then

$$
Y_{w}:=\overline{N_{w} \cdot o}=w X_{w}
$$

is a Schubert variety of type $w$.

By work of Kostant [9], the $\ell$-th exterior power $\wedge^{\ell} \mathfrak{g}_{-1}$ decomposes into irreducible $\mathfrak{g}_{0^{-}}$ modules $\mathbf{I}_{w}$, which are indexed by elements of $W^{\mathfrak{p}}$ of length $\ell$

$$
\Lambda^{\ell} \mathfrak{g}_{-1}=\bigoplus_{\substack{w \in W^{\mathfrak{p}} \\|w|=\ell}} \mathbf{I}_{w} .
$$

The highest weight line in $\mathbb{P} \mathbf{I}_{w}$ is $\mathfrak{n}_{w}$. Let $1 \in W^{\mathfrak{p}}$ be the identity, and let $w_{0} \in W^{\mathfrak{p}}$ be the longest element. Then $Y_{1}=o$ and $Y_{w_{0}}=X$, and $\mathbf{I}_{1}$ and $\mathbf{I}_{w_{0}}$ are trivial $\mathfrak{g}_{0}$-modules. Assume $w \in W^{\mathfrak{p}} \backslash\left\{1, w_{0}\right\}$. Let $\mathfrak{q}_{w} \subset \mathfrak{g}_{0}$ be the stabilizer of the highest weight line $\mathfrak{n}_{w}$. Then there is a subset $\mathrm{J}(w) \subset\{1, \ldots, r\} \backslash\{\mathbf{i}\}$ with the property that the Lie algebra $\mathfrak{q}_{w}$ is given by $\mathfrak{q}_{w}=\mathfrak{g}_{0, \geq 0}$, where

$$
\mathfrak{g}_{k, \ell}:=\left\{A \in \mathfrak{g}_{k} \mid\left[Z_{w}, A\right]=\ell A\right\} \quad \text { and } \quad Z_{w}:=\sum_{\mathrm{j} \in \mathrm{J}(w)} Z_{\mathrm{j}}
$$

We call $\mathfrak{g}=\oplus \mathfrak{g}_{k, \ell}$ the $\left(Z_{\mathfrak{i}}, Z_{w}\right)$-bigraded decomposition of $\mathfrak{g}$. It is a simple consequence of standard representation theory that $k \ell<0$ forces $\mathfrak{g}_{k, \ell}=0$. The following is $[15$, Proposition 3.9].

Proposition $2.7([15])$. Let $w \in W^{\mathfrak{p}} \backslash\left\{1, w_{0}\right\}$. There exists an integer $\mathrm{a}=\mathrm{a}(w) \geq 0$ such that the inversion set is given by

$$
\Delta(w)=\left\{\alpha \in \Delta\left(\mathfrak{g}_{1}\right) \mid \alpha\left(Z_{w}\right) \leq \mathrm{a}\right\} .
$$

Equivalently,

$$
\mathfrak{n}_{w}=\mathfrak{g}_{-1,0} \oplus \cdots \oplus \mathfrak{g}_{-1,-\mathbf{a}} .
$$


Remark 2.9. (a) By [15, Proposition 3.19], the Schubert variety $Y_{w}$ is smooth if and only if $\mathrm{a}(w)=0$. In particular, the proposition generalizes the characterization of the smooth Schubert varieties of $X$ by connected Dynkin sub-diagrams containing the $i$-th node. For more on the relationship between the integer $\mathrm{a}(w)$ and $\operatorname{Sing}\left(Y_{w}\right)$, see Corollary 3.6 .

(b) By (2.8b), the pair a $(w), \mathrm{J}(w)$ characterizes $\mathfrak{n}_{w}$ as a direct sum of $Z_{w}$-eigenspaces. This is the computational tool that we will use to work with the inversion set $\Delta(w)$.

(c) In the case that $X$ is a classical (types ABCD), well-known descriptions of Schubert varieties are given by partitions. Appendix A provides a 'translation' between the $(\mathrm{a}, \mathrm{J})$ and partition descriptions.

(d) The (a, J)-values for the Schubert classes in the two non-classical cominuscule varieties are given by Figures 1 and 2 .

(e) Since $\xi_{w}=\left[Y_{w}\right]$, and $Y_{w}$ is determined by $\Delta(w)$, the pair a $(w), \mathrm{J}(w)$ characterizes $\xi_{w}$, when $w \in W^{\mathfrak{p}} \backslash\left\{1, w_{0}\right\}$.

(f) A tableau-esque analog of Proposition 2.7 is given by H. Thomas and A. Yong in [16, Proposition 2.1].

Remark 2.10. I follow the notation of [15], with the following exception. In [15], we uniformly write $\mathrm{J}=\left\{j_{1}<\cdots<j_{\mathrm{p}}\right\}$. Here, it is convenient to reorder the $j_{\ell}$ in some cases.

\section{Singular LOCUS}

For this section we fix, once and for all, $w \in W^{\mathfrak{p}} \backslash\left\{1, w_{0}\right\}$. The singular locus $\operatorname{Sing}\left(X_{w}\right)$ is a union of Schubert subvarieties $X_{w^{\prime}} \subset X_{w}$. Let $\operatorname{Sing}_{w} \subset W^{\mathfrak{p}}$ be the subset indexing the irreducible components of $\operatorname{Sing}\left(X_{w}\right)$, so that

$$
\operatorname{Sing}\left(X_{w}\right)=\bigcup_{w^{\prime} \in \operatorname{Sing}_{w}} X_{w^{\prime}}
$$

Definition. Let $\Pi_{1, \mathrm{a}-1}:=\left\{\varepsilon \in \Delta\left(\mathfrak{g}_{1, \mathrm{a}-1}\right) \mid \varepsilon+\alpha \notin \Delta \forall \alpha \in \Delta^{+}\left(\mathfrak{g}_{0,0}\right)\right\}$. Equivalently, $\Pi_{1, \mathrm{a}-1}$ is the set of highest weights associated with the $\mathfrak{g}_{0,0}-$ module $\mathfrak{g}_{1, \mathrm{a}-1}$.

Let $\varepsilon \in \Pi_{1, a-1}$. Define

$$
\begin{aligned}
\Delta(w, \varepsilon) & :=\{\varepsilon\} \sqcup\left\{\nu \in \Delta\left(\mathfrak{g}_{1, a}\right) \mid \nu-\varepsilon \in \Delta\left(\mathfrak{g}_{0,1}\right)\right\} \\
& =\{\varepsilon\} \sqcup\left\{\Delta \cap\left(\varepsilon+\Delta\left(\mathfrak{g}_{0,1}\right)\right)\right\} .
\end{aligned}
$$

Lemma 3.2. There exists $w_{\varepsilon} \in W^{\mathfrak{p}}$ such that $\Delta\left(w_{\varepsilon}\right)=\Delta(w) \backslash \Delta(w, \varepsilon)$.

Theorem 3.3. The roots $\Pi_{1, a-1}$ are in bijective correspondence with the irreducible components of the singular locus $\operatorname{Sing}\left(X_{w}\right)$. Explicitly,

$$
\operatorname{Sing}_{w}=\left\{w_{\varepsilon} \mid \varepsilon \in \Pi_{1, \mathrm{a}-1}\right\} .
$$

The lemma and theorem are proved in Section 4.2.

Example. Theorem 3.3 generalizes the well-known descriptions [1, Section 9.3] of the singular locus of $X_{w}$ in the case that $G$ is classical. Here is a simple illustration in the case that $X=\operatorname{Gr}(5,11)$ is a Grassmannian. Assume the notations and definitions of Section A.4. In 
particular, the subalgebra $\mathfrak{g}_{-1} \subset \mathfrak{s l}_{n} \mathbb{C}$ is spanned by $\left\{e_{k}^{j} \mid 1 \leq j \leq 5,6 \leq k \leq 11\right\}$. The basis element $e_{k+1}^{j} \in \mathfrak{g}_{-1}$ is a root vector for the root

$$
-\alpha_{j k}:=-\left(\alpha_{j}+\cdots+\alpha_{k}\right) .
$$

Fix $w \in W^{\mathfrak{p}}$ with $\mathrm{a}(w)=2$ and $\mathrm{J}(w)=\{2,3,6,8,10\}$. Any element $s_{j}^{k} e_{k}^{j}$ of $\mathfrak{g}_{-1}$ may be represented by a matrix $\left(s_{k}^{j}\right)$ where $1 \leq j \leq 5$ and $6<k \leq 11$. The corresponding $Z_{w}$-degrees are given by

$$
-\left[\begin{array}{ll|l|ll}
2 & 2 & 1 & 0 & 0 \\
\hline 3 & 3 & 2 & 1 & 1 \\
3 & 3 & 2 & 1 & 1 \\
\hline 4 & 4 & 3 & 2 & 2 \\
4 & 4 & 3 & 2 & 2 \\
\hline 5 & 5 & 4 & 3 & 3
\end{array}\right]
$$

So the subspace $\mathfrak{n}_{w} \subset \mathfrak{g}_{-1}$ is represented by

$$
\mathfrak{n}_{w}=\left(\begin{array}{cc|c|cc}
s_{1}^{6} & s_{2}^{6} & s_{3}^{6} & s_{4}^{6} & s_{5}^{6} \\
\hline 0 & 0 & s_{3}^{7} & s_{4}^{7} & s_{5}^{7} \\
0 & 0 & s_{3}^{8} & s_{4}^{8} & s_{5}^{8} \\
\hline 0 & 0 & 0 & s_{4}^{9} & s_{5}^{9} \\
0 & 0 & 0 & s_{4}^{10} & s_{5}^{10} \\
\hline 0 & 0 & 0 & 0 & 0
\end{array}\right) .
$$

Define a filtration $F^{3} \subset F^{6} \subset F^{10} \subset \mathbb{C}^{11}$ by $F^{3}=\left\langle e_{1}, e_{2}, e_{6}\right\rangle, F^{6}=\left\langle F^{3}, e_{3}, e_{7}, e_{8}\right\rangle$ and $F^{10}=\left\langle F^{6}, e_{4}, e_{5}, e_{9}, e_{10}\right\rangle$. Then $(2.5)$ yields $X_{w}=w^{-1} Y_{w}$, with

$$
Y_{w}=\left\{E \in \operatorname{Gr}(5,11) \mid \operatorname{dim}\left(E \cap F^{3}\right) \geq 2, \operatorname{dim}\left(E \cap F^{6}\right) \geq 3, \operatorname{dim}\left(E \cap F^{10}\right) \geq 5\right\} .
$$

The subalgebra $\mathfrak{g}_{0,0}$ is identified with the diagonal block matrices $\operatorname{diag}(2,1,2,1,2,2,1)$ in $\mathfrak{s l}_{11}$. The $\mathfrak{g}_{0,0}-$ module $\mathfrak{g}_{-1,-1}$ consists of two irreducible submodules. The first is spanned by $e_{6}^{3}$, with highest weight $\varepsilon_{1}=-\alpha_{35}$. The second is four-dimensional with highest weight vector $e_{8}^{4}$ and highest weight $\varepsilon_{2}=-\alpha_{47}$. We have $\Delta\left(w, \varepsilon_{1}\right)=\left\{\alpha_{15}, \alpha_{25}, \alpha_{35}, \alpha_{36}, \alpha_{37}\right\}$ and $\Delta\left(w, \varepsilon_{2}\right)=\left\{\alpha_{37}, \alpha_{47}, \alpha_{48}, \alpha_{49}\right\} . *$ The two irreducible components $X_{w_{1}}=w_{1}^{-1} Y_{w_{1}}$ and $X_{w_{2}}=w_{2}^{-1} Y_{w_{2}}$ of $\operatorname{Sing}\left(X_{w}\right)$ correspond to

$$
\mathfrak{n}_{w_{1}}=\left(\begin{array}{cc|c|cc}
0 & 0 & 0 & s_{4}^{6} & s_{5}^{6} \\
\hline 0 & 0 & 0 & s_{4}^{7} & s_{5}^{7} \\
0 & 0 & 0 & s_{4}^{8} & s_{5}^{8} \\
\hline 0 & 0 & 0 & s_{4}^{9} & s_{5}^{9} \\
0 & 0 & 0 & s_{4}^{10} & s_{5}^{10} \\
\hline 0 & 0 & 0 & 0 & 0
\end{array}\right) \quad \text { and } \quad \mathfrak{n}_{w_{2}}=\left(\begin{array}{cc|c|cc}
s_{1}^{6} & s_{2}^{6} & s_{3}^{6} & s_{4}^{6} & s_{5}^{6} \\
\hline 0 & 0 & s_{3}^{7} & s_{4}^{7} & s_{5}^{7} \\
0 & 0 & 0 & s_{4}^{8} & s_{5}^{8} \\
\hline 0 & 0 & 0 & 0 & s_{5}^{9} \\
0 & 0 & 0 & 0 & s_{5}^{10} \\
\hline 0 & 0 & 0 & 0 & 0
\end{array}\right) .
$$

The corresponding a and $\mathrm{J}$ values are $\mathrm{a}\left(w_{1}\right)=0$ and $\mathrm{J}\left(w_{2}\right)=\{3,10\} ;$ and $\mathrm{a}\left(w_{2}\right)=2$ and $\mathrm{J}_{w_{2}}=\{2,4,6,7,10\}$.

*Note that the associated subspaces of $\mathfrak{n}_{w}$ are 'hooks' that are added to $\pi$ to obtain the partitions associated with the irreducible components of $\operatorname{Sing}\left(X_{\pi}\right)$. 
Corollary 3.4. The Schubert variety $X_{w}$ is smooth if and only if $\mathrm{a}(w)=0$. If $\mathrm{a}(w)=1$, then $\operatorname{Sing}\left(X_{w}\right)$ is a single irreducible Schubert variety.

Remark. The first part of the corollary was proved in [15, Proposition 3.9].

Proof. The first statement is an immediate consequence of $\mathfrak{g}_{1, a-1}=\mathfrak{g}_{1,-1}=\{0\}$. The second statement is a consequence of the fact that $\mathfrak{g}_{1, a-1}=\mathfrak{g}_{1,0}$ is an irreducible $\mathfrak{g}_{0,0}$-module, see [15, Section 3.2].

The following is an immediate consequence of Theorem 3.3.

Corollary 3.5. The number of irreducible components in $\operatorname{Sing}\left(X_{w}\right)$ is the number $\left|\Pi_{1, \mathrm{a}-1}\right|$ of components in a decomposition of $\mathfrak{g}_{1, a-1}$ into irreducible $\mathfrak{g}_{0,0}$-submodules.

From Corollary 3.5, and the (a, J)-characterizations of Sections A.4-A.6 we deduce

Corollary 3.6. Let $X_{w}$ be a Schubert variety in a classical, irreducible cominuscule $X=$ $G / P$, and let $\left|\operatorname{Sing}_{w}\right|$ be the number of irreducible components in $\operatorname{Sing}\left(X_{w}\right)$.

(a) If $X=\operatorname{Gr}(\mathbf{i}, n+1)$, then $\left|\operatorname{Sing}_{w}\right|=\mathrm{a}(w)$.

(b) If $X=\mathrm{LG}(n, 2 n)$, then $\left|\operatorname{Sing}_{w}\right|=\lceil\mathrm{a}(w) / 2\rceil$.

(c) Suppose $X=\mathcal{S}_{n}$ and assume $\mathrm{a}(w)>1$. Set $\mathrm{r}=\left\lceil\frac{1}{2}\left(\mathrm{a}+\alpha_{n-1}\left(Z_{w}\right)\right)\right\rceil$. If $1=\mathrm{j}_{\mathrm{r}-1}-\mathrm{j}_{\mathrm{r}}$, then $\left|\operatorname{Sing}_{w}\right|=\left\lfloor\frac{1}{2}\left(\mathrm{a}+\alpha_{n-1}\left(Z_{w}\right)\right)\right\rfloor \in\{\mathbf{r}-1, \mathrm{r}\}$; otherwise $\left|\operatorname{Sing}_{w}\right|=\mathbf{r}$.

Remark. The singular loci of Schubert varieties in quadric hypersurfaces $B_{n} / P_{1}$ and $D_{n} / P_{1}$ are so simple that I omitted them from the corollary. See [1, Section 9.3].

Proof of Corollary 3.6(a). Adopt the notation of Appendix A.4, and assume a $>0$. Then the highest $\mathfrak{g}_{0,0}$-weights of $\mathfrak{g}_{1, \mathrm{a}-1}$ are

$$
\varepsilon=\alpha_{j_{\ell}+1}+\cdots+\alpha_{i}+\cdots+\alpha_{\mathrm{k}_{m}-1},
$$

with $0<\ell, m$ and $\ell+m=\mathrm{a}+1$. Thus $\left|\Pi_{1, \mathrm{a}-1}\right|=\mathrm{a}(w)$.

Proof of Corollary 3.6(b). Adopt the notation of Appendix A.4, and assume a $>0$. Suppose that $\mathrm{a}=2 \mathrm{~s}$. Then the highest $\mathfrak{g}_{0,0}$-weights of $\mathfrak{g}_{1, \mathrm{a}-1}$ are

$$
\varepsilon=\alpha_{j_{m}+1}+\cdots+\alpha_{j_{\ell}}+2\left(\alpha_{j_{\ell}+1}+\cdots+\alpha_{n-1}\right)+\alpha_{n}
$$

with $1 \leq \ell<m$ and $\ell+m=\mathrm{a}+1=2 \mathrm{~s}+1$. Therefore, $\left|\Pi_{1, \mathrm{a}-1}\right|=\mathrm{s}=\lceil\mathrm{a}(w) / 2\rceil$.

If $\mathrm{a}=2 \mathrm{~s}-1$, then the highest $\mathfrak{g}_{0,0}-$ weights of $\mathfrak{g}_{1, \mathrm{a}-1}$ are $(*)$ and

$$
\varepsilon=2\left(\alpha_{j_{s}+1}+\cdots+\alpha_{n-1}\right)+\alpha_{n} .
$$

Thus, $\left|\Pi_{1, \mathrm{a}-1}\right|=\mathrm{s}=\lceil\mathrm{a}(w) / 2\rceil$.

The proof of Corollary 3.7(c), which is very similar to, though more tedious than, that of Corollary $3.7(\mathrm{~b})$, is left to the reader.

3.1. The exceptional cases. The irreducible components of $\operatorname{Sing}\left(X_{w}\right)$ have been determined in the case that $G$ is classical and $P$ is (co)minuscule; see [1, Section 9.3]. A fourth corollary of Theorem 3.3 is an explicit description of the singular locus of the Schubert varieties in the exceptional Cayley plane $E_{6} / P_{6}$ and Freudenthal variety $E_{7} / P_{7}$ (both of which are minuscule and cominuscule). See Tables 1 and 2 on pages 8 and 9 , respectively. The tables are obtained with the assistance of [11]. 
Key to Tables 1 and 2. Each row represents a proper $(\neq o, X)$ Schubert variety $X_{w}$ of $X$, indexed by $w \in W^{\mathfrak{p}} \backslash\left\{1, w_{0}\right\}$. The first column is the dimension of $X_{w}$; the second column expresses $w$ as a reduced product of simple reflections, acting on the left; the third column gives the corresponding $\mathrm{a}(w): \mathrm{J}(w)$ values, see Section 2.2; and the fourth column lists the irreducible components of the singular locus in terms of their a : J characterization. (See also Figures 1 and 2 on pages 21 and 22, respectively.)

Remarks. From the tables, we see that:

(a) The the irreducible components $\left\{X_{w_{\varepsilon}} \mid \varepsilon \in \Pi_{1, a-1}\right\}$ of the singular locus of a Schubert variety $X_{w}$ in $E_{6} / P_{6}$ or $E_{7} / P_{7}$ satisfy $\operatorname{codim}_{X_{w}} X_{w_{\varepsilon}} \geq 3$.

(b) The singular locus of a Schubert variety in the Cayley plane consists of at most one irreducible component.

(c) The singular locus of a Schubert variety in the Freudenthal variety consists of at most two irreducible components.

TABLE 1. Schubert varieties and their singular loci in the Cayley plane.

\begin{tabular}{|c|l|l|l|}
\hline $\operatorname{dim}$ & $w$ & $\mathrm{a}: \mathrm{J}$ & $\operatorname{Sing}_{w}$ \\
\hline \hline 1 & 6 & $0: 5$ & \\
\hline 2 & 65 & $0: 4$ & \\
\hline 3 & 654 & $0: 23$ & \\
\hline 4 & 6542 & $0: 3$ & \\
\hline 4 & 6543 & $0: 12$ & \\
\hline 5 & 65432 & $1: 123$ & $0: 4$ \\
\hline 5 & 65431 & $0: 2$ & \\
\hline 6 & 654321 & $1: 23$ & $0: 4$ \\
\hline 6 & 654324 & $1: 14$ & $0: 5$ \\
\hline 7 & 6543241 & $2: 124$ & $0: 3$ \\
\hline 7 & 6543245 & $1: 15$ & $o$ \\
\hline 8 & 65432413 & $1: 4$ & $0: 5$ \\
\hline 8 & 65432451 & $2: 125$ & $0: 3$ \\
\hline
\end{tabular}

\begin{tabular}{|c|l|l|l|}
\hline $\operatorname{dim}$ & $w$ & $\mathrm{a}: \mathrm{J}$ & $\operatorname{Sing}_{w}$ \\
\hline \hline 8 & 65432456 & $0: 1$ & \\
\hline 9 & 654324513 & $3: 145$ & $1: 23$ \\
\hline 9 & 654324561 & $1: 12$ & $0: 3$ \\
\hline 10 & 6543245134 & $2: 35$ & $0: 2$ \\
\hline 10 & 6543245613 & $2: 14$ & $1: 23$ \\
\hline 11 & 65432451342 & $1: 5$ & $o$ \\
\hline 11 & 65432456134 & $3: 135$ & $1: 4$ \\
\hline 12 & 654324561342 & $2: 15$ & $1: 4$ \\
\hline 12 & 654324561345 & $1: 3$ & $0: 2$ \\
\hline 13 & 6543245613452 & $3: 35$ & $2: 14$ \\
\hline 14 & 65432456134524 & $2: 4$ & $1: 12$ \\
\hline 15 & 654324561345243 & $1: 2$ & $0: 1$ \\
\hline
\end{tabular}

3.2. Codimension of the singular locus. Let $\varepsilon \in \Pi_{1, \mathrm{a}-1}$. By Lemma 3.2 and Theorem 3.3 , the irreducible component $X_{w_{\varepsilon}} \subset \operatorname{Sing}\left(X_{w}\right)$ has codimension $|\Delta(w, \varepsilon)|$. In this section we characterize the Schubert varieties $X_{w}$ for which the codimension is minimal. Zelevinskiu [17] showed that every Schubert variety in the Grassmannian admits a small resolution. So, in particular, we know that $\operatorname{codim}_{X_{w}} X_{w_{\varepsilon}} \geq 3$ for all $X_{w} \subset \operatorname{Gr}(i, n+1)$. This inequality also holds (and is sharp) for Schubert varieties in the spinor variety $\mathcal{S}_{n}=D_{n} / P_{n}$. On the other hand, the Lagrangian Grassmannian admits Schubert varieties with codim $X_{w} X_{w_{\varepsilon}}=2$. 
TABLE 2. Schubert varieties and their singular loci in the Freudenthal variety.

\begin{tabular}{|c|l|l|l|}
\hline $\operatorname{dim}$ & $w$ & $\mathrm{a}: \mathrm{J}$ & Sing $_{w}$ \\
\hline \hline 1 & 7 & $0: 6$ & \\
\hline 2 & 76 & $0: 5$ & \\
\hline 3 & 765 & $0: 4$ & \\
\hline 4 & 7654 & $0: 23$ & \\
\hline 5 & 76542 & $0: 3$ & \\
\hline 5 & 76543 & $0: 12$ & \\
\hline 6 & 765432 & $1: 123$ & $0: 4$ \\
\hline 6 & 765431 & $0: 2$ & \\
\hline 7 & 7654321 & $1: 23$ & $0: 4$ \\
\hline 7 & 7654324 & $1: 14$ & $0: 5$ \\
\hline 8 & 76543241 & $2: 124$ & $0: 3$ \\
\hline 8 & 76543245 & $1: 15$ & $0: 6$ \\
\hline 9 & 765432413 & $1: 4$ & $0: 5$ \\
\hline 9 & 765432451 & $2: 125$ & $0: 3$ \\
\hline 9 & 765432456 & $1: 16$ & $o$ \\
\hline 10 & 7654324513 & $3: 145$ & $1: 23$ \\
\hline 10 & 7654324561 & $2: 126$ & $0: 3$ \\
\hline 10 & 7654324567 & $0: 1$ & \\
\hline 11 & 76543245134 & $2: 35$ & $0: 2$ \\
\hline 11 & 76543245613 & $3: 146$ & $1: 23$ \\
\hline 11 & 76543245671 & $1: 12$ & $0: 3$ \\
\hline 12 & 765432451342 & $1: 5$ & $0: 6$ \\
\hline & & & \\
\hline
\end{tabular}

\begin{tabular}{|c|l|l|l|}
\hline $\operatorname{dim}$ & $w$ & $\mathrm{a}: \mathrm{J}$ & Sing $_{w}$ \\
\hline \hline 12 & 765432456134 & $4: 1356$ & $1: 4$ \\
\hline 12 & 765432456713 & $2: 14$ & $1: 23$ \\
\hline 13 & 7654324561342 & $3: 156$ & $1: 4$ \\
\hline 13 & 7654324561345 & $2: 36$ & $0: 2$ \\
\hline 13 & 7654324567134 & $3: 135$ & $1: 4$ \\
\hline 14 & 76543245613452 & $4: 356$ & $3: 146$ \\
\hline 14 & 76543245671342 & $2: 15$ & $1: 4$ \\
\hline 14 & 76543245671345 & $3: 136$ & $2: 35$ \\
\hline 15 & 765432456134524 & $3: 46$ & $2: 126$ \\
\hline 15 & 765432456713452 & $5: 1356$ & $1: 5,2: 14$ \\
\hline 15 & 765432456713456 & $1: 3$ & $0: 2$ \\
\hline 16 & 7654324561345243 & $2: 26$ & $1: 16$ \\
\hline 16 & 7654324567134524 & $4: 146$ & $1: 5,1: 12$ \\
\hline 16 & 7654324567134562 & $3: 35$ & $2: 14$ \\
\hline 17 & 76543245613452431 & $1: 6$ & $o$ \\
\hline 17 & 76543245671345243 & $3: 126$ & $1: 5,0: 1$ \\
\hline 17 & 76543245671345624 & $5: 346$ & $2: 15$ \\
\hline 18 & 765432456713452431 & $2: 16$ & $1: 5$ \\
\hline 18 & 765432456713456243 & $4: 236$ & $2: 15$ \\
\hline 18 & 765432456713456245 & $2: 4$ & $1: 12$ \\
\hline 19 & 7654324567134562431 & $3: 36$ & $2: 15$ \\
\hline 19 & 7654324567134562453 & $5: 246$ & $3: 35$ \\
\hline
\end{tabular}

\begin{tabular}{|l|l|l|l|}
\hline $\operatorname{dim}$ & $w$ & $\mathrm{a}: \mathrm{J}$ & $\operatorname{Sing}_{w}$ \\
\hline \hline 20 & 76543245671345624531 & $4: 46$ & $3: 35$ \\
\hline 20 & 76543245671345624534 & $3: 25$ & $1: 3$ \\
\hline 21 & 765432456713456245341 & $5: 256$ & $2: 4$ \\
\hline 21 & 765432456713456245342 & $1: 2$ & $0: 1$ \\
\hline 22 & 7654324567134562453421 & $3: 26$ & $2: 4$ \\
\hline 22 & 7654324567134562453413 & $2: 5$ & $1: 3$ \\
\hline 23 & 76543245671345624534132 & $4: 25$ & $4: 46$ \\
\hline 24 & 765432456713456245341324 & $3: 4$ & $3: 36$ \\
\hline 25 & 7654324567134562453413245 & $2: 3$ & $2: 16$ \\
\hline 26 & 76543245671345624534132456 & $1: 1$ & $1: 6$ \\
\hline
\end{tabular}

Corollary 3.7. Let $X=G / P$ be cominuscule. Fix $w \in W^{\mathfrak{p}} \backslash\left\{1, w_{0}\right\}$ with associated a, J. Let $\varepsilon \in \Pi_{1, \mathrm{a}-1}$. 
(a) Suppose $X=\operatorname{Gr}(\mathrm{i}, n+1)=A_{n} / P_{\mathrm{i}}$. Then $\operatorname{codim}_{X_{w}} X_{w_{\varepsilon}} \geq 3$. Assume the notation of Section A.4. Equality holds if and only if there exist $0<\ell \leq \mathrm{p}$ and $0<m \leq \mathrm{q}$ such that $\ell+m=\mathrm{a}+1$ and $1=\mathrm{j}_{\ell}-\mathrm{j}_{\ell+1}=\mathrm{k}_{m+1}-\mathrm{k}_{m}$.

(b) Suppose $X=\operatorname{LG}(n, 2 n)=C_{n} / P_{n}$. Then $\operatorname{codim}_{X_{w}} X_{w_{\varepsilon}} \geq 2$. Assume the notation of Section A.5. Equality holds if and only if $\mathrm{a}=2 \ell-1>0$ and $1=j_{\ell}-j_{\ell+1}$. In particular, these $X_{w}$ admit no small resolution.

(c) Suppose $X=\mathcal{S}_{n}=D_{n} / P_{n}$. Then $\operatorname{codim}_{X_{w}} X_{w_{\varepsilon}} \geq 3$. Assume the notation of Section A.6. If $\mathrm{j}_{1}=n-1$ and $\mathrm{a}=1$, then equality holds if and only if $\mathrm{j}_{2}=n-3$; if $\mathrm{j}_{1}=n-1$ and $\mathrm{a}=2$, then equality holds if and only if $\mathrm{j}_{2}=n-2$ and $\mathrm{j}_{3}=n-4$. In all other cases, equality holds if and only if there exist $\alpha_{n-1}\left(Z_{w}\right)<\ell<m$ such that $\ell+m=\mathrm{a}+1+\alpha_{n-1}\left(Z_{w}\right)$, and $1+\delta_{\ell \mathrm{r}}=\mathrm{j}_{\ell}-\mathrm{j}_{\ell+1}$ and $1=\mathrm{j}_{m}-\mathrm{j}_{m+1}$.

Proof of Corollary 3.7(a). The elements of $\Pi_{1, a-1}$ are of the form

$$
\varepsilon=\alpha_{j_{\ell}+1}+\cdots+\alpha_{\mathrm{k}_{m}-1}, \quad \text { with } \ell+m=\mathrm{a}+1 \text { and } 0 \leq \ell, m .
$$

Both $\nu_{1}=\alpha_{j_{\ell}}$ and $\nu_{2}=\alpha_{\mathrm{k}_{m}}$ are elements of $\Delta\left(\mathfrak{g}_{0,1}\right)$, and $\varepsilon, \varepsilon+\nu_{1}$ and $\varepsilon+\nu_{2}$ are distinct elements of $\Delta(w, \varepsilon)$. Thus $\operatorname{codim}_{X_{w}} X_{w_{\varepsilon}}=|\Delta(w, \varepsilon)| \geq 3$. Additionally, $|\Delta(w, \varepsilon)|=3$ if and only if $1=\mathrm{j}_{\ell}-\mathrm{j}_{\ell+1}=\mathrm{k}_{m+1}-\mathrm{k}_{m}$.

Proof of Corollary 3.7(b). The elements of $\Pi_{1, a-1}$ are of the form $\varepsilon^{\prime}=\alpha_{j_{a}+1}+\cdots+\alpha_{n}$, or

$$
\varepsilon=\alpha_{j_{\ell}+1}+\cdots+\alpha_{j_{m}}+2\left(\alpha_{j_{m}+1}+\cdots+\alpha_{n-1}\right)+\alpha_{n},
$$

with $\ell+m=\mathrm{a}+1$ and $0<m \leq \ell$. For $\varepsilon^{\prime}$, observe that $\nu_{1}=\alpha_{\mathrm{j}_{\mathrm{a}}} \in \Delta\left(\mathfrak{g}_{0,1}\right)$ and $\varepsilon^{\prime}, \varepsilon^{\prime}+\nu_{1} \in \Delta\left(w, \varepsilon^{\prime}\right)$. So $\operatorname{codim}_{X_{w}} X_{w_{\varepsilon}^{\prime}}=\left|\Delta\left(w, \varepsilon^{\prime}\right)\right| \geq 2$. Equality holds if and only if $1=j_{\mathrm{a}}-\mathrm{j}_{\mathrm{a}+1}$ and $\mathrm{a}=1$. (If $\mathrm{a}>1$, then $\nu_{2}=\alpha_{\mathrm{j}_{1}}+\cdots+\alpha_{n-1} \in \Delta\left(\mathfrak{g}_{0,1}\right)$ and $\varepsilon^{\prime}+\nu_{2} \in \Delta(w, \varepsilon)$ is distinct from $\varepsilon^{\prime}$ and $\varepsilon^{\prime}+\nu_{1}$.)

For $\varepsilon$ with $\ell<m$, observe that both $\nu_{1}=\alpha_{j_{\ell}}$ and $\nu_{2}=\alpha_{j_{m}}$ are elements of $\Delta\left(\mathfrak{g}_{0,1}\right)$, and $\varepsilon, \varepsilon+\nu_{1}$ and $\varepsilon+\nu_{2}$ are distinct elements of $\Delta(w, \varepsilon)$. Thus $\operatorname{codim}_{X_{w}} X_{w_{\varepsilon}}=|\Delta(w, \varepsilon)| \geq 3$. If $\ell=m$, then $\nu_{1}=\nu_{2}$. So, $\operatorname{codim}_{X_{w}} X_{w_{\varepsilon}}=|\Delta(w, \varepsilon)| \geq 2$. Equality holds if and only if $1=\mathrm{j}_{\ell}-\mathrm{j}_{\ell+1}$.

The proof of Corollary 3.7(c), which is very similar to, though more involved than, that of Corollary $3.7(\mathrm{~b})$, is left to the reader.

\section{Proof of Theorem 3.3}

4.1. The stabilizer of $\boldsymbol{Y}_{\boldsymbol{w}}$. Virtue of Proposition 4.1, the stabilizer $\operatorname{Stab}\left(Y_{w}\right)=\{g \in$ $\left.G \mid g Y_{w}=Y_{w}\right\}$ will play an important rôle in the proof of Theorem 3.3.

Proposition 4.1 (Brion-Polo [4]). The smooth locus $Y_{w}^{0}$ of $Y_{w}$ is the orbit of $o \in X$ under the stabilizer $G_{w}$.

In this section we will apply Proposition 2.7 to obtain a description of the stabilizer in terms of the the data $(\mathrm{a}(w), \mathrm{J}(w))$. Review the definitions of the grading elements $Z_{\mathrm{i}}$ and $Z_{w}$, and the $\left(Z_{i}, Z_{w}\right)$-bigraded decomposition $\mathfrak{g}=\oplus \mathfrak{g}_{j, k}$, in Sections 2.1 and 2.2.

Lemma 4.2. The largest subalgebra $\mathfrak{g}_{w} \subset \mathfrak{g}$ containing $\mathfrak{n}_{w}$ and such that $\mathfrak{g}_{w} \equiv \mathfrak{n}_{w}$ mod $\mathfrak{g}_{\geq 0}$ is

$$
\mathfrak{g}_{w}=\mathfrak{n}_{w} \oplus \mathfrak{g}_{0, \geq 0} \oplus \mathfrak{g}_{1, \geq a} .
$$


It is well-known that the stabilizer of the Schubert variety $Y_{w}$ is parabolic; see, for example, [1] or [4]. The following is a corollary of Lemma 4.2.

Corollary 4.4. The stabilizer of $Y_{w}$ in $G$ is the parabolic subgroup $G_{w}$ associated with $\mathfrak{g}_{w}$.

Proof of Lemma 4.2. Let $\mathfrak{g}^{\prime}$ denote the right-hand side of (4.3). It is clear that $\mathfrak{g}^{\prime}$ is a subalgebra of $\mathfrak{g}$, and that $\mathfrak{g}^{\prime} \equiv \mathfrak{n}_{w} \bmod \mathfrak{g}_{\geq 0}$. So $\mathfrak{g}^{\prime} \subset \mathfrak{g}_{w}$. By construction $\mathfrak{g}_{0, \geq 0}$ is the stabilizer of $\mathfrak{n}_{w}$ in $\mathfrak{g}_{0}$, see Section 2.2. Consequently, $\mathfrak{g}_{w} \equiv \mathfrak{n}_{w} \oplus \mathfrak{g}_{0, \geq 0} \bmod \mathfrak{g}_{1}$. So it remains to see that,

$$
\mathfrak{g}_{1,<\mathrm{a}} \cap \mathfrak{g}_{w}=0 .
$$

Assume the converse: suppose there exists a nonzero a nonzero $\zeta \in \mathfrak{g}_{1,<a} \cap \mathfrak{g}_{w}$. Since $\mathfrak{g}_{1}$ is an irreducible $\mathfrak{g}_{0}-$ module, and $\mathfrak{g}_{0, \geq 0} \oplus \mathfrak{g}_{1, \geq a} \subset \mathfrak{g}_{w}$, we may assume without loss of generality that $\zeta \in \mathfrak{g}_{1, \mathrm{a}-1}$. Then $\left[\zeta, \mathfrak{n}_{w}\right] \subset \mathfrak{g}_{w}$ holds if and only if $\left[\zeta, \mathfrak{g}_{-1,-\mathrm{a}}\right]=0$. In particular, $U=\left\{\zeta \in \mathfrak{g}_{1, \mathrm{a}-1} \mid\left[\zeta, \mathfrak{g}_{-1,-\mathrm{a}}\right]=0\right\} \neq 0$.

The Jacobi identity implies $U$ is a $\mathfrak{g}_{0,0}$-module. So there exists $\Delta(U) \subset \Delta\left(\mathfrak{g}_{1, \mathrm{a}-1}\right)$ such that $U=\oplus_{\alpha \in \Delta(U)} \mathfrak{g}_{\alpha}$. Let $\gamma \in \Delta(U)$ be a highest $\mathfrak{g}_{0,0}$-weight. Let $\tilde{\alpha}$ be the highest root of $\mathfrak{g}$. Then there exists a sequence $\sigma_{1}, \ldots, \sigma_{\ell} \in \Sigma$ of simple roots such that $\gamma_{i}:=\tilde{\gamma}-\sigma_{1}-\cdots-\sigma_{i}$ is a root, for each $1 \leq i \leq \ell$, and $\gamma_{\ell}=\gamma$. Since $\gamma$ is a highest $\mathfrak{g}_{0,0}-$ weight, and $\gamma_{\ell}+\sigma_{\ell}=\gamma_{\ell-1}$ is a root, $\sigma_{\ell}$ must lie in $\Delta\left(\mathfrak{g}_{0,1}\right)$. In particular, $\gamma_{\ell-1} \in \Delta\left(\mathfrak{g}_{1, \mathrm{a}}\right)$. From $\gamma-\gamma_{\ell-1}=-\sigma_{\ell}$, we see that $0 \neq \mathfrak{g}_{-\sigma_{\ell}}=\left[\mathfrak{g}_{\gamma}, \mathfrak{g}_{-\gamma_{\ell-1}}\right] \subset\left[\mathfrak{g}_{\gamma}, \mathfrak{g}_{-1,-\mathrm{a}}\right]$, implying $\mathfrak{g}_{\gamma} \not \subset U$, a contradiction. We conclude that (4.5) must hold.

The algebra $\mathfrak{g}_{w}$ is a nonstandard parabolic; that is, $\mathfrak{g}_{w}$ does not contain the fixed Borel subalgebra $\mathfrak{b} \subset \mathfrak{g}$. Nonetheless, it admits a useful description in terms of the element

$$
\tilde{Z}_{w}=Z_{w}-\mathrm{a} Z_{\mathfrak{i}} \in \mathfrak{h} .
$$

As an element of the Cartan subalgebra, $\tilde{Z}_{w}$ acts on $\mathfrak{g}$ by eigenvalues. Set $\mathrm{t}=\max \left\{\alpha\left(\tilde{Z}_{w}\right) \mid \alpha \in\right.$ $\Delta$ \}. The $\tilde{Z}_{w}$-graded decomposition of $\mathfrak{g}$ is the eigenspace decomposition

$$
\mathfrak{g}=\bigoplus_{s=-\mathrm{t}}^{\mathrm{t}} \tilde{\mathfrak{g}}_{s} \quad \text { where } \quad \tilde{\mathfrak{g}}_{s}:=\left\{\zeta \in \mathfrak{g} \mid\left[\tilde{Z}_{w}, \zeta\right]=s \zeta\right\}
$$

Given (4.3), it is straight-forward to confirm that

$$
\mathfrak{g}_{w}=\tilde{\mathfrak{g}}_{\geq 0} .
$$

(Note that this provides a second proof that $\mathfrak{g}_{w}$ is parabolic, cf. [5, Theorem 3.2.1(2)].)

Remark 4.7. We see from (4.6) that the pair $(\mathrm{a}, \mathrm{J})$ encodes the relationship between the two parabolic subalgebras $\mathfrak{p}$ and $\mathfrak{g}_{w}$. Moreover, (2.6) and (4.6) yield $\mathfrak{g}_{0,0}=\mathfrak{g}_{0} \cap \tilde{\mathfrak{g}}_{0}$ and $\mathfrak{g}_{1, \mathrm{a}-1}=\mathfrak{g}_{1} \cap \tilde{\mathfrak{g}}_{-1}$. So Corollary 3.5 may be interpreted as saying the following: to the pair of parabolic subalgebras $\mathfrak{p}=\mathfrak{g}_{\geq 0}$ and $\mathfrak{g}_{w}=\tilde{\mathfrak{g}}_{\geq 0}$ is naturally associated a reductive subalgebra $\mathfrak{r}=\mathfrak{g}_{0} \cap \tilde{\mathfrak{g}}_{0}$ and a $\mathfrak{r}-$ module $U=\mathfrak{g}_{1} \cap \tilde{\mathfrak{g}}_{-1}$, such that the irreducible components of the singular locus of $Y_{w}$ are in bijection with the irreducible $\mathfrak{r}$-submodules of $U$. 
4.2. Lemmas. We begin with a proof of Lemma 3.2. The remainder of the section is then devoted to the proof of Theorem 3.3; the theorem is an immediate corollary of Lemmas 4.9 and 4.11 .

Remark 4.8. One important consequence of Remark 2.3 is that given a set $\Phi \subset \Delta\left(\mathfrak{g}_{1}\right)$, there exists $w \in W^{\mathfrak{p}}$ such that $\Delta(w)=\Phi$ if and only if $\Delta^{+} \backslash \Phi$ is closed. For details see [15, Section 2.3].

Proof of Lemma 3.2. By Remark 2.3 it suffices to show that

$$
\begin{aligned}
\Phi(w, \varepsilon):=\Delta^{+} \backslash\{\Delta(w) \backslash \Delta(w, \varepsilon)\} & =\left\{\Delta^{+} \backslash \Delta(w)\right\} \sqcup \Delta(w, \varepsilon) \\
& =\Delta\left(\mathfrak{g}_{1,>a}\right) \sqcup \Delta^{+}\left(\mathfrak{g}_{0, \geq 0}\right) \sqcup \Delta(w, \varepsilon)
\end{aligned}
$$

is closed. By Remark 2.3, the set $\Delta\left(\mathfrak{g}_{1,>\mathrm{a}}\right) \sqcup \Delta(w, \varepsilon) \subset \Delta\left(\mathfrak{g}_{1}\right)$ is closed. Similarly, by Remark 4.8, the set $\Delta^{+} \backslash \Delta(w)=\Delta\left(\mathfrak{g}_{1,>\mathrm{a}}\right) \sqcup \Delta^{+}\left(\mathfrak{g}_{0, \geq 0}\right)$ is closed. So it remains to show that given roots $\nu \in \Delta(w, \varepsilon)$ and $\beta \in \Delta^{+}\left(\mathfrak{g}_{0, \geq 0}\right)$ such that $\nu+\beta$ is also a root, it is the case that $\nu+\beta \in \Phi(w, \varepsilon)$. There are two cases to consider: either $\nu=\varepsilon$, or $\nu=\varepsilon+\nu^{\prime}$ for some $\nu^{\prime} \in \Delta\left(\mathfrak{g}_{0,1}\right)$.

(I) Assume $\nu=\varepsilon$ and $\nu+\beta \in \Delta$.

○ If $\beta \in \Delta\left(\mathfrak{g}_{0,>1}\right)$, then $\nu+\beta \in \Delta\left(\mathfrak{g}_{1,>\mathrm{a}}\right) \subset \Phi(w, \varepsilon)$.

$\circ$ If $\beta \in \Delta\left(\mathfrak{g}_{0,1}\right)$, then $\nu+\beta \in \Delta(w, \varepsilon) \subset \Phi(w, \varepsilon)$.

○ If $\beta \in \Delta^{+}\left(\mathfrak{g}_{0,0}\right)$, then $\nu+\beta=\varepsilon+\beta$ cannot be a root because $\varepsilon$ is a highest $\mathfrak{g}_{0,0}$-weight.

(II) Assume $\nu=\varepsilon+\nu^{\prime} \in \Delta\left(\mathfrak{g}_{1, \mathrm{a}}\right)$ and $\nu+\beta \in \Delta$.

○ If $\beta \in \Delta\left(\mathfrak{g}_{0,>0}\right)$, then $\nu+\beta \in \Delta\left(\mathfrak{g}_{1,>\mathrm{a}}\right) \subset \Phi(w, \varepsilon)$.

○If $\beta \in \Delta^{+}\left(\mathfrak{g}_{0,0}\right)$, then $\nu+\beta=\varepsilon+\nu^{\prime}+\beta \in \Delta$; therefore,

$$
\begin{aligned}
\{0\} \neq \mathfrak{g}_{\nu+\beta} & =\left[\mathfrak{g}_{\nu}, \mathfrak{g}_{\beta}\right]=\left[\left[\mathfrak{g}_{\varepsilon}, \mathfrak{g}_{\nu^{\prime}}\right], \mathfrak{g}_{\beta}\right] \\
& =\left[\left[\mathfrak{g}_{\beta}, \mathfrak{g}_{\nu^{\prime}}\right], \mathfrak{g}_{\varepsilon}\right]+\left[\left[\mathfrak{g}_{\varepsilon}, \mathfrak{g}_{\beta}\right], \mathfrak{g}_{\nu^{\prime}}\right] .
\end{aligned}
$$

Because $\varepsilon$ is a highest $\mathfrak{g}_{0,0}$-weight, the bracket $\left[\mathfrak{g}_{\varepsilon}, \mathfrak{g}_{\beta}\right]$ is zero. This forces $\left[\mathfrak{g}_{\beta}, \mathfrak{g}_{\nu^{\prime}}\right]$ to be nonzero. Equivalently, $\beta+\nu^{\prime} \in \Delta\left(\mathfrak{g}_{0,1}\right)$. Thus $\nu+\beta=\varepsilon+\left(\nu^{\prime}+\beta\right) \in \Delta(w, \varepsilon)$.

Lemma 4.9. The set $\left\{\Delta\left(w_{\varepsilon}\right) \mid \varepsilon \in \Pi_{1, \mathrm{a}-1}\right\}$ is precisely the collection of $\Delta\left(w_{1}\right) \subset \Delta(w)$, with $w_{1} \in W^{\mathfrak{p}}$, that are maximal with the property that

$$
\Delta(w) \backslash \Delta\left(w_{1}\right) \not \subset \Delta\left(\mathfrak{g}_{1, \mathrm{a}}\right) .
$$

Lemma 4.11. Given $w_{1}<w$, we have $X_{w_{1}} \subset \operatorname{Sing}\left(X_{w}\right)$ if and only if $\Delta(w) \backslash \Delta\left(w_{1}\right) \not \subset$ $\Delta\left(\mathfrak{g}_{1, \mathrm{a}}\right)$.

Given a root $\gamma$, let $r_{\gamma} \in W$ denote the associated reflection. In order to prove Lemmas 4.9 and 4.11 we first recall

Lemma 4.12. Let $w_{1}, w \in W^{\mathfrak{p}}$ be elements of the Hasse diagram of a cominuscule $G / P$. Then $w_{1} \leq w$ if and only if $\Delta\left(w_{1}\right) \subset \Delta(w)$. In this case there is an ordering $\left\{\gamma_{1}, \gamma_{2}, \ldots, \gamma_{m}\right\}$ of the elements of $\Delta(w) \backslash \Delta\left(w_{1}\right)$ so that $w_{\ell+1}=r_{\gamma_{\ell}} w_{\ell} \in W^{\mathfrak{p}}, w_{m+1}=w$ and $\Delta\left(w_{\ell+1}\right)=$ $\Delta\left(w_{1}\right) \sqcup\left\{\gamma_{1}, \ldots, \gamma_{\ell}\right\}$. 
Proof. This well-known result may be deduced from Propositions 3.2.12(5) and 3.2.15(3) of [5]. Note that their $\Phi_{w}$ is our $\Delta(w)$.

Corollary 4.13. The Weyl group element $w_{1}=r_{\varepsilon} w_{\varepsilon}$ is an element of $W^{\mathfrak{p}}$ and $\Delta\left(r_{\varepsilon} w_{\varepsilon}\right)=$ $\Delta\left(w_{\varepsilon}\right) \sqcup\{\varepsilon\}$. Moreover, there exists an ordering $\left\{\nu_{1}, \cdots, \nu_{m}\right\}$ of the elements of $\Delta(w, \varepsilon) \backslash\{\varepsilon\}$ so that $w_{\ell+1}:=r_{\nu_{\ell}} \cdots r_{\nu_{1}} w_{1} \in W^{\mathfrak{p}}$ and $\Delta\left(w_{\ell+1}\right)=\Delta\left(w_{\varepsilon}\right) \sqcup\left\{\varepsilon, \nu_{1}, \ldots, \nu_{\ell}\right\}$, for all $1 \leq \ell \leq m$.

Proof. It suffices to observe that, in the ordering of the $\operatorname{roots} \Delta(w, \varepsilon)=\Delta(w) \backslash \Delta\left(w_{\varepsilon}\right)$ given by Lemma 4.12, it is necessarily the case that $\gamma_{1}=\varepsilon$. By Remark 4.8, the set $\Phi=\Delta^{+} \backslash \Delta\left(r_{\gamma_{1}} w_{\varepsilon}\right)$ is closed. Suppose that $\gamma_{1} \neq \varepsilon$. Then, by the definition (3.1) of $\Delta(w, \varepsilon)$, there exists $\mu \in \Delta\left(\mathfrak{g}_{0,1}\right)$ such that $\gamma_{1}=\varepsilon+\mu$. However, $\varepsilon, \mu \in \Phi$, while $\gamma_{1} \notin \Phi$, contradicting the closure of $\Phi$.

Proof of Lemma 4.9. Recall (Proposition 2.7) that $\Delta(w)=\Delta\left(\mathfrak{g}_{1,<\mathrm{a}}\right)$. By Lemma 3.2, $\Delta(w) \backslash \Delta\left(w_{\varepsilon}\right)=\Delta(w, \varepsilon)$. The definition (3.1) yields $\Delta(w, \varepsilon) \cap \Delta\left(\mathfrak{g}_{1,<\mathrm{a}}\right)=\{\varepsilon\}$. So $\Delta(w, \varepsilon) \not \subset$ $\Delta\left(\mathfrak{g}_{1, \mathrm{a}}\right)$. To see that $\Delta\left(w_{\varepsilon}\right)$ is maximal with respect to (4.10), recall (Remark 4.8) that $\Delta^{+} \backslash \Delta\left(w_{\varepsilon}\right)$ is closed; this forces $\Delta(w, \varepsilon) \subset \Delta^{+} \backslash \Delta\left(w_{\varepsilon}\right)$.

Conversely, suppose that $\Delta\left(w_{1}\right) \subset \Delta(w)$ satisfies (4.10). Fix $\mu=\mu_{0} \in \Delta\left(\mathfrak{g}_{1,<\mathrm{a}}\right) \backslash \Delta\left(w_{1}\right)$. There exists a sequence of simple roots $\sigma_{1}, \ldots, \sigma_{\ell} \in \Sigma$ such that each $\mu_{i}:=\mu+\sigma_{1}+\cdots+\sigma_{i}$ is a root, for all $1 \leq i \leq \ell$, and $\mu_{\ell}$ is the highest root of $\mathfrak{g}$. Since both $\mu$ and $\mu_{\ell}$ lie in $\Delta\left(\mathfrak{g}_{1}\right)$, the simple roots $\sigma_{i}$ must lie in $\Delta^{+}\left(\mathfrak{g}_{0}\right)$. It follows from Remark 4.8 that each $\mu_{i} \in \Delta^{+} \backslash \Delta\left(w_{1}\right)$. Moreover, since $\mu_{\ell}$ is the highest root of $\mathfrak{g}$, at least one of the $\mu_{i}$ is an element of $\Delta\left(\mathfrak{g}_{1, a-1}\right)$. Let $U \subset \mathfrak{g}_{1, a-1}$ be the irreducible $\mathfrak{g}_{0,0}$-submodule containing $\mathfrak{g}_{\mu_{i}}$. Let $\varepsilon \in \Delta(U)$ be the highest $\mathfrak{g}_{0,0}$-weight of $U$. There exists a second sequence of simple roots $\sigma_{1}^{\prime}, \ldots, \sigma_{m}^{\prime} \in \Sigma\left(\mathfrak{g}_{0,0}\right)$ such that each $\mu_{i, k}:=\mu_{i}+\sigma_{1}^{\prime}+\cdots+\sigma_{k}^{\prime} \in \Delta(U)$, with $1 \leq k \leq m$, and $\mu_{i, m}=\varepsilon$. Since $\mu_{i} \in \Delta^{+} \backslash \Delta\left(w_{1}\right)$, Remark 4.8 implies $\varepsilon \in \Delta^{+} \backslash \Delta\left(w_{1}\right)$. As in the first paragraph of this proof, Remark 4.8 forces $\Delta(w, \varepsilon) \subset \Delta^{+} \backslash \Delta\left(w_{1}\right)$. Thus, $\Delta\left(w_{1}\right) \subset \Delta\left(w_{\varepsilon}\right)$.

Proof of Lemma 4.11. First we will show that the lemma is equivalent to (4.14c). Recall from (2.5) that $Y_{w}=w X_{w}$. So the lemma is equivalent to

$$
w X_{w_{1}} \subset \operatorname{Sing}\left(Y_{w}\right) \quad \text { if and only if } \quad \Delta(w) \backslash \Delta\left(w_{1}\right) \not \subset \Delta\left(\mathfrak{g}_{1, \mathrm{a}}\right) .
$$

By Lemma 4.12 we have $w=\tau w_{1}$, where $\tau=r_{\gamma_{m}} \cdots r_{\gamma_{2}} r_{\gamma_{1}}$ and $\left\{\gamma_{1}, \gamma_{2}, \ldots, \gamma_{m}\right\}=$ $\Delta(w) \backslash \Delta\left(w_{1}\right)$. So $w X_{w_{1}}=\tau Y_{w_{1}}$, and (4.14a) is equivalent to

$$
\tau Y_{w_{1}} \subset \operatorname{Sing}\left(Y_{w}\right) \quad \text { if and only if } \Delta(w) \backslash \Delta\left(w_{1}\right) \not \subset \Delta\left(\mathfrak{g}_{1, \mathrm{a}}\right) .
$$

By Lemma 4.12 we have $\Delta\left(w_{1}\right) \subset \Delta(w)$. Equations (2.4) and (4.3) then imply $\mathfrak{n}_{w_{1}} \subset \mathfrak{n}_{w} \subset$ $\mathfrak{g}_{w}$, and therefore $N_{w_{1}} \subset N_{w} \subset G_{w}$. By Proposition 4.1, $G_{w} \cdot o=Y_{w} \backslash \operatorname{Sing}\left(Y_{w}\right)=Y_{w}^{0}$. So $N_{w_{1}} \cdot o \subset G_{w} \cdot o=Y_{w}^{0}$. Since $Y_{w_{1}}=\overline{N_{w_{1}} \cdot o}$, we see that $\tau Y_{w_{1}} \subset \operatorname{Sing}\left(Y_{w}\right)$ if and only if $\tau N_{w_{1}} \cdot o \not \subset G_{w} \cdot o$. Therefore, (4.14b) is equivalent to

$$
\tau N_{w_{1}} \cdot o \not \subset G_{w} \cdot o \quad \text { if and only if } \Delta(w) \backslash \Delta\left(w_{1}\right) \not \subset \Delta\left(\mathfrak{g}_{1, \mathrm{a}}\right) .
$$

Let $\gamma \in \Delta(w) \backslash \Delta\left(w_{1}\right)$. As an element of $W=N_{G}(H) / H$, the reflection $r_{\gamma}$ is represented by $\exp (\xi) \exp (\zeta) \exp (\xi) \in N_{G}(H)$, where the $\xi \in \mathfrak{g}_{\gamma}$ and $\zeta \in \mathfrak{g}_{-\gamma}$ are scaled so that $\gamma([\xi, \zeta])=$ -2 ; see, for example, the proof of [5, Theorem 3.2.19(1)]. Lemma 4.2 and Corollary 4.4 imply that

$$
r_{\gamma} \in G_{w} \quad \text { if and only if } \quad \gamma \in \Delta\left(\mathfrak{g}_{1, \mathrm{a}}\right)
$$


In this case, $r_{\gamma} N_{w_{1}} \subset r_{\gamma} G_{w}=G_{w}$. This establishes one direction of (4.14c): if $\Delta(w) \backslash \Delta\left(w_{1}\right) \subset$ $\Delta\left(\mathfrak{g}_{1, \mathrm{a}}\right)$, then $\tau \in G_{w}$ and $\tau N_{w_{1}} \cdot o \subset G_{w} \cdot o=Y_{w} \backslash \operatorname{Sing}\left(Y_{w}\right)$.

Suppose $\Delta(w) \backslash \Delta\left(w_{1}\right) \not \subset \Delta\left(\mathfrak{g}_{1, \mathrm{a}}\right)$. By Lemma 4.9 there exists $\varepsilon \in \Pi_{1, \mathrm{a}-1}$ such that $\Delta\left(w_{1}\right) \subset \Delta\left(w_{\varepsilon}\right)$.

Claim. If $r_{\varepsilon} \notin G_{w} P$, then $\tau N_{w_{1}} \cdot o \not \subset G_{w} \cdot o$.

Assume that claim holds. Then to establish the second direction of (4.14c), it remains to show that the reflection $r_{\varepsilon} \in W$ can not be represented by an element $\tilde{p} p \in G_{w} P$ with $\tilde{p} \in G_{w}$ and $p \in P$.

Recall the $Z_{\mathfrak{i}}$-graded decomposition (2.1) of $\mathfrak{g}$. Let $G_{0}:=\left\{g \in G \mid \operatorname{Ad}_{g}\left(\mathfrak{g}_{j}\right) \subset \mathfrak{g}_{j}\right\}$. Then $G_{0}$ is a closed subgroup of $G$ with Lie algebra $\mathfrak{g}_{0}$. By [5, Theorem 3.1.3], the map $\mathfrak{g}_{1} \times G_{0} \rightarrow P$ sending $(u, g) \mapsto \exp (u) g$ is a diffeomorphism. Likewise, recall the $\widetilde{Z}_{w}$-graded decomposition (4.6b) of $\mathfrak{g}$. Again, $\widetilde{G}_{0}:=\left\{g \in G \mid \operatorname{Ad}_{g}\left(\tilde{\mathfrak{g}}_{k}\right) \subset \tilde{\mathfrak{g}}_{k}\right\}$ is a closed subgroup of $G$ with Lie algebra $\tilde{\mathfrak{g}}_{0}$. By (4.6c), $\mathfrak{g}_{w}=\tilde{\mathfrak{g}}_{\geq 0}=\tilde{\mathfrak{g}}_{0} \oplus \tilde{\mathfrak{g}}_{+}$, and [5, Theorem 3.1.3] implies that the map $\tilde{\mathfrak{g}}_{+} \times \widetilde{G}_{0} \rightarrow G_{w}$ sending $(\tilde{u}, \tilde{g}) \mapsto \exp (\tilde{u}) \tilde{g}$ is a diffeomorphism.

We will argue by contradiction, supposing that $\tilde{p} p \in G_{w} P$ represents the reflection $r_{\varepsilon}$. In particular, $\operatorname{Ad}_{\tilde{p} p}: \mathfrak{g} \rightarrow \mathfrak{g}$ preserves the Cartan subalgebra $\mathfrak{h}$. Write $\tilde{p}=\exp (\tilde{u}) \tilde{g}$, with $\tilde{u} \in \tilde{\mathfrak{g}}_{+}$and $\tilde{g} \in \widetilde{G}_{0}$, and $p=\exp (u) g$, with $g \in G_{0}$ and $u \in \mathfrak{g}_{1}$. Fix $H \in \mathfrak{h}$, and define $h_{s} \in \tilde{\mathfrak{g}}_{s}$ by $\operatorname{Ad}_{p} H=\sum_{s=-\mathrm{t}}^{\mathrm{t}} \tilde{h}_{s}$, and set $\tilde{h}_{s}=\operatorname{Ad}_{\tilde{g}} h_{s} \in \tilde{\mathfrak{g}}_{s}$. Define $\tilde{h}_{s, r} \in \tilde{\mathfrak{g}}_{r}$ by $\operatorname{Ad}_{\exp (\tilde{u})} \tilde{h}_{s}=\tilde{h}_{s, s}+\tilde{h}_{s, s+1}+\cdots+\tilde{h}_{s, \mathrm{t}}$, and note that $\tilde{h}_{s, s}=\tilde{h}_{s}$. Then

$$
\operatorname{Ad}_{\tilde{p} p} H=\sum_{s=-\mathrm{t}}^{\mathrm{t}} \sum_{r=s}^{\mathrm{t}} \tilde{h}_{s, r}=\sum_{r=-\mathrm{t}}^{\mathrm{t}} \tilde{H}_{r},
$$

where $\tilde{H}_{r}:=\sum_{s=-\mathrm{t}}^{r} \tilde{h}_{s, r} \in \tilde{\mathfrak{g}}_{r}$.

Since $\operatorname{Ad}_{\tilde{p} p}$ preserves $\mathfrak{h}$, and $\mathfrak{h} \subset \tilde{\mathfrak{g}}_{0}$, it must be the case that

$$
\tilde{H}_{0} \in \mathfrak{h}, \quad \text { and } \quad \tilde{H}_{r}=0 \text {, when } r \neq 0 .
$$

In particular, $\tilde{H}_{-\mathrm{t}}=\tilde{h}_{-\mathrm{t},-\mathrm{t}}=\tilde{h}_{-\mathrm{t}}=0$. This in turn yields $\tilde{h}_{-\mathrm{t}, r}=0$ for all $r$. Moreover, since $\tilde{h}_{-\mathrm{t}}=\operatorname{Ad}_{\tilde{g}} h_{-\mathrm{t}}$, we also have $h_{-\mathrm{t}}=0$. Next, $0=\tilde{H}_{1-\mathrm{t}}=\tilde{h}_{-\mathrm{t}, 1-\mathrm{t}}+\tilde{h}_{1-\mathrm{t}, 1-\mathrm{t}}=\tilde{h}_{1-\mathrm{t}}$. As above, this implies $\tilde{h}_{1-\mathrm{t}, r}=0$, for all $r$, and $h_{1-\mathrm{t}}=0$. Continuing by induction, we see that

$$
h_{s}=0 \text { for all } s<0 .
$$

In particular, $\operatorname{Ad}_{p} H \in \tilde{\mathfrak{g}}_{>0}$. Our choice of $H \in \mathfrak{h}$ was arbitrary, so $\operatorname{Ad}_{p} \mathfrak{h} \subset \tilde{\mathfrak{g}}_{>0}=\mathfrak{g}_{w}$. This implies $p \in G_{w}$. In particular, $\tilde{p} p \in G_{w}$.

This yields a contradiction as follows. Note that $\mathfrak{g}_{-\varepsilon} \subset \tilde{\mathfrak{g}}_{1}$. So given any $q \in G_{w}$, we have $\operatorname{Ad}_{q} \mathfrak{g}_{-\varepsilon} \subset \tilde{\mathfrak{g}}_{\geq 1}$. On the other hand, $\mathfrak{g}_{\varepsilon} \subset \tilde{\mathfrak{g}}_{-1}$, and $\operatorname{Ad}_{r_{\varepsilon}}\left(\mathfrak{g}_{-\varepsilon}\right)=\mathfrak{g}_{\varepsilon}$. Therefore, there exists no element $q \in G_{w}$ such that $\operatorname{Ad}_{q}\left(\mathfrak{g}_{-\varepsilon}\right)=\tilde{\mathfrak{g}}_{\varepsilon}$. Modulo the claim, this completes the proof of Lemma 4.11.

Proof of claim. By Lemma 4.12, $w_{1} \leq w_{\varepsilon}$. Therefore, $X_{w_{1}} \subset X_{w_{\varepsilon}}$. So to see that $X_{w_{1}} \subset$ $\operatorname{Sing}\left(X_{w}\right)$, it suffices to show that $X_{w_{\varepsilon}} \subset \operatorname{Sing}\left(X_{w}\right)$. Equivalently, as discussed above, $\tau N_{w_{\varepsilon}} \cdot o \not \subset G_{w} \cdot o$, where $\tau=r_{\nu_{m}} \cdots r_{\nu_{1}} r_{\varepsilon}$ is as given by Corollary 4.13. Since $\nu_{\ell} \in \Delta\left(\mathfrak{g}_{1, \mathrm{a}}\right)$, we have $r_{\nu_{\ell}} \in G_{w}$, by (A.11). So $\tau N_{w_{\varepsilon}} \cdot o \not \subset G_{w} \cdot o$ if and only if $r_{\varepsilon} N_{w_{\varepsilon}} \cdot o \not \subset G_{w} \cdot o$. In particular, to see that $\tau N_{w_{\varepsilon}} \cdot o \not \subset G_{w} \cdot o$, it suffices to show that $r_{\varepsilon} \cdot o \notin G_{w} \cdot o$. Lifting to $G$, the latter is equivalent to $r_{\varepsilon} \notin G_{w} P$. 


\section{Appendix A. Geometric Descriptions of $Y_{\mathrm{a}, \mathrm{J}}$}

The classical cominuscule $G / P$ admit geometric, partition based descriptions. A 'dictionary' relating these descriptions to the representation theoretic $(\mathrm{a}, \mathrm{J})$-description (Section 2.2 ) is given in [14, Appendix A]. We now briefly summarize those results for the reader's convenience.

A.1. Notation. Given a vector space $V \simeq \mathbb{C}^{m}$, we fix a basis $\left\{e_{1}, \ldots, e_{m}\right\}$. Let $\left\{e^{1}, \ldots, e^{m}\right\}$ denote the dual basis of $V^{*}$. Set

$$
e_{\ell}^{k} \stackrel{\text { dfn }}{=} e_{\ell} \otimes e^{k} \in \operatorname{End}(V) \text { for all } \leq k, \ell \leq m .
$$

- When $V \simeq \mathbb{C}^{2 n+1}$ is of odd dimension and admits a nondegenerate symmetric bilinear form $(\cdot, \cdot)$, then we will normalize the basis so that $\left(e_{k}, e_{\ell}\right)=\left(e_{n+k}, e_{n+\ell}\right)=\left(e_{k}, e_{2 n+1}\right)=$ $\left(e_{n+k}, e_{2 n+1}\right)=0,\left(e_{k}, e_{n+\ell}\right)=\delta_{k \ell}$, for all $1 \leq k, \ell \leq n$, and $\left(e_{2 n+1}, e_{2 n+1}\right)=1$.

- When $V \simeq \mathbb{C}^{2 n}$ is of even dimension and admits a nondegenerate (symmetric or skewsymmetric) bilinear form $(\cdot, \cdot)$, we normalize the basis so that $\left(e_{k}, e_{\ell}\right)=\left(e_{n+k}, e_{n+\ell}\right)=0$ and $\left(e_{k}, e_{n+\ell}\right)=\delta_{k \ell}$, for all $1 \leq k, \ell \leq n$. In this setting, we fix an isotropic flag $F^{\bullet}$ in $\mathbb{C}^{2 n}$ by specifying $F^{k}=\left\langle e_{1}, \ldots, e_{k}\right\rangle$ and $\left(F^{k}, F^{2 n-k}\right)=0$ for $1 \leq k \leq n$.

A.2. Odd dimensional quadrics $\boldsymbol{Q}^{2 n-1}=\boldsymbol{B}_{n} / \boldsymbol{P}_{\mathbf{1}}$. Set $m=2 n-1$. There is a bijection between $W^{\mathfrak{p}} \backslash\left\{1, w_{0}\right\}$ and pairs $\mathrm{a}, \mathrm{J}$ such that $\mathrm{J}=\{\mathrm{j}\} \subset\{2, \ldots, n\}$ and $\mathrm{a} \in\{0,1\}$; see $[15$, Corollary 3.17]. If $\mathrm{a}=0$, then

$$
Y_{w}=\mathbb{P}\left\langle e_{1}, \ldots, e_{j}\right\rangle=\mathbb{P}^{j-1} .
$$

If $\mathrm{a}=1$, then

$$
Y_{w}=Q^{m} \cap \mathbb{P}\left\langle e_{1}, \ldots, e_{n+1}, e_{n+j+1}, \ldots, e_{2 n+1}\right\rangle .
$$

A.3. Even dimensional quadrics $\boldsymbol{Q}^{\mathbf{2 n - 2}}=\boldsymbol{D}_{n} / \boldsymbol{P}_{\mathbf{1}}$. Set $m=2 n-2$. There is a bijection between $W^{\mathfrak{p}} \backslash\left\{1, w_{0}\right\}$ and pairs a, J such that either

$\circ \mathrm{a}=0$ and $\mathrm{J}=\{\mathrm{j}\} \subset\{2, \ldots, n\}$ or $\mathrm{J}=\{n-1, n\}$; or

$\circ \mathrm{a}=1$ and $\mathrm{J}=\{\mathrm{j}\} \subset\{2, \ldots, n-2\}$ or $\mathrm{J}=\{n-1, n\}$.

See [15, Corollary 3.17]. First suppose that $\mathrm{a}=0$. If $\mathrm{J}=\{\mathrm{j}\}$ with $2 \leq \mathrm{j} \leq n-2$, then $X_{w}=\mathbb{P}^{\mathrm{j}-1}$. If $\mathrm{J}=\{n-1\}$ or $\mathrm{J}=\{n\}$, then $X_{w}=\mathbb{P}^{n-1}$. If $\mathrm{J}=\{n-1, n\}$, then $X_{w}=\mathbb{P}^{n-2}$.

Next suppose that $\mathrm{a}=1$. If $\mathrm{J}=\{\mathrm{j}\}$ with $2 \leq \mathrm{j} \leq n-2$, then $X_{w}=Q^{m} \cap$ $\mathbb{P}\left\langle e_{1}, \ldots, e_{n+1}, e_{n+j+1}, \ldots, e_{2 n}\right\rangle$. If $\mathrm{J}=\{n-1, n\}$, then $X_{w}=Q^{m} \cap \mathbb{P}\left\langle e_{1}, \ldots, e_{n+1}, e_{2 n}\right\rangle$.

A.4. Grassmannians $\operatorname{Gr}(i, n+1)=A_{n} / P_{\mathfrak{i}}$. There is a bijection between $W^{\mathfrak{p}} \backslash\left\{1, w_{0}\right\}$ and pairs a, $\mathrm{J}$ such that $\mathrm{J}=\left\{\mathrm{j}_{\mathrm{p}}, \ldots, \mathrm{j}_{1}, \mathrm{k}_{1}, \ldots, \mathrm{k}_{\mathrm{q}}\right\} \subset\{1, \ldots, n\} \backslash\{\mathrm{i}\}$ is ordered so that

$$
1 \leq \mathrm{j}_{\mathrm{p}}<\cdots<\mathrm{j}_{1}<\mathrm{i}<\mathrm{k}_{1}<\cdots<\mathrm{k}_{\mathrm{q}} \leq n
$$

and satisfying $\mathrm{p}, \mathrm{q} \in\{\mathrm{a}, \mathrm{a}+1\}$; see [15, Corollary 3.17]. (Beware, these $\mathrm{p}, \mathrm{q}$ do not agree with those of [15], cf. Remark 2.10.) For convenience we set

$$
\mathrm{j}_{\mathrm{p}+1}:=0, \quad \mathrm{j}_{0}:=\mathrm{i}=: \mathrm{k}_{0}, \quad \mathrm{k}_{\mathrm{q}+1}:=n+1 .
$$

It is well-known that Schubert varieties in $X=\operatorname{Gr}(i, n+1)$ are indexed by partitions

$$
\lambda=\left(\lambda_{1}, \ldots, \lambda_{\dot{i}}\right) \in \mathbb{Z}^{\mathrm{i}} \text { such that } 1 \leq \lambda_{1}<\lambda_{2}<\cdots<\lambda_{\mathrm{i}} \leq n+1,
$$


cf. $[1, \S 3.1 .3]$. Fix a flag $0 \subset F^{1} \subset F^{2} \subset \cdots \subset F^{n+1}$. The corresponding Schubert variety is

$$
Y_{\lambda}\left(F^{\bullet}\right):=\left\{E \in X \mid \operatorname{dim}\left(E \cap F^{\lambda_{k}}\right) \geq k, \forall k\right\} .
$$

Note that, if $\lambda_{k+1}=\lambda_{k}+1$, then the condition $\operatorname{dim}\left(E \cap F^{\lambda_{k}}\right) \geq k$ is redundant; it is implied by $\operatorname{dim}\left(E \cap F^{\lambda_{k+1}}\right) \geq k+1$. To remove the redundancies, decompose $\lambda=\mu_{\mathrm{p}} \cdots \mu_{1} \mu_{0}$ into maximal blocks of consecutive integers. For example, if $\lambda=(2,3,4,7,8,12)$, then $\mu_{2}=(2,3,4), \mu_{1}=(7,8)$ and $\mu_{0}=(12)$. Let

$$
\mathrm{j}_{\ell}(\lambda)=\left|\mu_{\mathrm{p}} \cdots \mu_{\ell}\right|
$$

be the length of the sub-partition $\mu_{\mathrm{p}} \cdots \mu_{\ell}$. (In all cases, $j_{0}=|\lambda|=i$.) The following is [15, Proposition 3.30].

Lemma A.4 ([15]). Let $\lambda=\left(\lambda_{1}, \ldots, \lambda_{i}\right)$ be a partition satisfying (A.1), and let $\lambda=$ $\mu_{\mathrm{p}} \cdots \mu_{1} \mu_{0}$ be the decomposition of $\lambda$ into maximal blocks of consecutive integers. The pair $\mathrm{a}, \mathrm{J}=\left\{\mathrm{j}_{\mathrm{p}}, \ldots, \mathrm{j}_{1}, \mathrm{k}_{1}, \ldots, \mathrm{k}_{\mathrm{q}}\right\}$ characterizing the Schubert variety $Y_{\lambda}$ is given by (A.3),

$$
\left\{\mathrm{k}_{1}, \ldots, \mathrm{k}_{\mathrm{q}}\right\}=\left\{\mathrm{i}-\mathrm{j}_{\mathrm{p}}+\lambda_{\mathrm{j}_{\mathrm{p}}}, \ldots, \mathrm{i}-\mathrm{j}_{1}+\lambda_{\mathrm{j}_{1}}, \lambda_{\mathrm{i}}\right\} \backslash\{\mathrm{i}, n+1\},
$$

and

$$
\mathrm{a}=\left\{\begin{array}{ll}
\mathrm{p} & \text { if } \lambda_{1}>1 \\
\mathrm{p}-1 & \text { if } \lambda_{1}=1
\end{array}\right\}= \begin{cases}\mathrm{q}, & \text { if } \lambda_{\mathrm{i}}=n+1 \\
\mathrm{q}-1, & \text { if } \lambda_{\mathrm{i}}<n+1\end{cases}
$$

Conversely, given $\mathrm{a}, \mathrm{J}$, the associated partition $\lambda=\mu_{\mathrm{p}} \cdots \mu_{1} \mu_{0}$ is given by

$$
\mu_{\ell}=\left(j_{\ell+1}+k_{m}-i+1, \ldots, j_{\ell}+k_{m}-i\right),
$$

with $\ell+m=\mathrm{a}+1$.

Example A.5. Consider $X=\operatorname{Gr}(5,13) \simeq A_{12} / P_{5}$. For the marking $\mathrm{J}=\{2,3,7,9,12\}$ and integer $\mathrm{a}=2$, we have $\lambda=(3,4,7,11,12)$.

A.5. Lagrangian Grassmannians $\mathrm{LG}(n, 2 n)=C_{n} / P_{n}$. There exists a bijection between $W^{\mathfrak{p}} \backslash\left\{1, w_{0}\right\}$ and pairs $\mathrm{a} \geq 0$ and $\mathrm{J}=\left\{\mathrm{j}_{\mathrm{p}}, \ldots, \mathrm{j}_{1}\right\} \subset\{1, \ldots, n-1\}$ satisfying

$$
1 \leq j_{p}<\cdots<j_{1} \leq n-1
$$

and

$$
\mathrm{p} \in\{\mathrm{a}, \mathrm{a}+1\} ;
$$

see $\left[15\right.$, Corollary 3.17]. (These $j_{\ell}$ have the opposite order of those in [15].) For convenience we set

$$
\mathrm{j}_{\mathrm{p}+1}:=0, \quad \mathrm{j}_{0}:=n .
$$

It is well-known that Schubert varieties in $X=\operatorname{LG}(n, 2 n)$ are indexed by partitions $\lambda=\left(\lambda_{1}, \lambda_{2}, \ldots, \lambda_{n}\right)$ such that

$$
\begin{aligned}
& 1 \leq \lambda_{1}<\lambda_{2}<\cdots<\lambda_{n} \leq 2 n, \quad \text { and } \\
& \lambda_{i} \in \lambda \text { if and only if } 2 n+1-\lambda_{i} \notin \lambda,
\end{aligned}
$$

cf. $[1, \S 9.3]$. The corresponding Schubert variety is given by (A.2), with $F^{\bullet}$ an isotropic flag as in Section A.1. 
Lemma A.9 ([14]). Let $\lambda=\left(\lambda_{1}, \ldots, \lambda_{n}\right)$ be a partition satisfying (A.8). Let $\lambda=\mu_{\mathrm{p}} \cdots \mu_{1} \mu_{0}$ be a decomposition of $\lambda$ into $\mathrm{p}+1$ maximal blocks of consecutive integers. Then $\mathrm{J}(\lambda)=$ $\left\{\mathrm{j}_{\mathrm{p}}(\lambda), \ldots, \mathrm{j}_{1}(\lambda)\right\}$ is given by (A.3), and

$$
\mathrm{a}(\lambda)= \begin{cases}\mathrm{p}-1 & \text { if } \lambda_{1}=1 \\ \mathrm{p} & \text { if } \lambda_{1}>1\end{cases}
$$

Conversely, given a and $\mathrm{J}=\left\{\mathrm{j}_{\mathrm{p}}, \cdots, \mathrm{j}_{1}\right\}$ we construct $\lambda(\mathrm{a}, \mathrm{J})=\mu_{\mathrm{p}}(\mathrm{a}, \mathrm{J}) \cdots \mu_{0}(\mathrm{a}, \mathrm{J})$ by

$$
\mu_{\ell}(\mathrm{a}, \mathrm{J})=\left(n+1+\mathrm{j}_{\ell+1}-\mathrm{j}_{m}, \ldots, n+\mathrm{j}_{\ell}-\mathrm{j}_{m}\right)
$$

with $\ell+m=\mathrm{a}+1$.

As an example, Table 3 lists the partitions $\lambda$ and corresponding a : $\mathrm{J}$ values for the Schubert varieties in $\mathrm{LG}(5,10)$.

TABLE 3. Schubert varieties of $\operatorname{LG}(5,10)$.

\begin{tabular}{|c|c||c|c||c|c|}
\hline$\lambda$ & $\mathrm{a}: \mathrm{J}$ & $\lambda$ & $\mathrm{a}: \mathrm{J}$ & $\lambda$ & $\mathrm{a}: \mathrm{J}$ \\
\hline \hline$(1,2,3,4,5)$ & & $(1,2,3,4,6)$ & $0: 4$ & $(1,2,3,5,7)$ & $1: 3,4$ \\
\hline$(1,2,4,5,8)$ & $1: 2,4$ & $(1,2,3,6,7)$ & $0: 3$ & $(1,3,4,5,9)$ & $1: 1,4$ \\
\hline$(1,2,4,6,8)$ & $2: 2,3,4$ & $(2,3,4,5,10)$ & $1: 4$ & $(1,3,4,6,9)$ & $2: 1,3,4$ \\
\hline$(1,2,5,7,8)$ & $1: 2,3$ & $(2,3,4,6,10)$ & $2: 3,4$ & $(1,3,5,7,9)$ & $3: 1,2,3,4$ \\
\hline$(1,2,6,7,8)$ & $0: 2$ & $(2,3,5,7,10)$ & $3: 2,3,4$ & $(1,4,5,8,9)$ & $1: 1,3$ \\
\hline$(1,3,6,7,9)$ & $2: 1,2,4$ & $(2,4,5,8,10)$ & $3: 1,3,4$ & $(2,3,6,7,10)$ & $2: 2,4$ \\
\hline$(1,4,6,8,9)$ & $2: 1,2,3$ & $(3,4,5,9,10)$ & $1: 3$ & $(2,4,6,8,10)$ & $4: 1,2,3,4$ \\
\hline$(1,5,7,8,9)$ & $1: 1,2$ & $(3,4,6,9,10)$ & $2: 2,3$ & $(2,5,7,8,10)$ & $3: 1,2,4$ \\
\hline$(1,6,7,8,9)$ & $0: 1$ & $(3,5,7,9,10)$ & $3: 1,2,3$ & $(2,6,7,8,10)$ & $2: 1,4$ \\
\hline$(4,5,8,9,10)$ & $1: 2$ & $(3,6,7,9,10)$ & $2: 1,3$ & $(4,6,8,9,10)$ & $2: 1,2$ \\
\hline$(5,7,8,9,10)$ & $1: 1$ & $(6,7,8,9,10)$ & & & \\
\hline
\end{tabular}

A.6. Spinor varieties $\mathcal{S}_{n}=D_{n} / P_{n}$. Given $\mathrm{a}=\mathrm{a}(w)$ and $\mathrm{J}=\mathrm{J}(w)$, note that

$$
\alpha_{n-1}\left(Z_{w}\right)=0 \text { if } n-1 \notin \mathrm{J}, \quad \text { and } \quad \alpha_{n-1}\left(Z_{w}\right)=1 \text { if } n-1 \in \mathrm{J} .
$$

Define

$$
\mathrm{r}=\left\lceil\frac{1}{2}\left(\mathrm{a}+\alpha_{n-1}\left(Z_{w}\right)\right)\right\rceil= \begin{cases}\lceil\mathrm{a} / 2\rceil & \text { if } n-1 \notin \mathrm{J} \\ \lfloor\mathrm{a} / 2\rfloor+1 & \text { if } n-1 \in \mathrm{J}\end{cases}
$$

There exists a bijection between $W^{\mathfrak{p}} \backslash\left\{1, w_{0}\right\}$, and pairs $\mathrm{a} \geq 0$ and $\mathrm{J}=\left\{\mathrm{j}_{\mathrm{p}}, \ldots, \mathrm{j}_{1}\right\} \subset$ $\{1, \ldots, n-1\}$, ordered by (A.6) and satisfying

$$
\mathrm{p}-\alpha_{n-1}\left(Z_{w}\right) \in\{\mathrm{a}, \mathrm{a}+1\}, \text { and } 2 \leq \mathrm{j}_{\mathrm{r}}-\mathrm{j}_{\mathrm{r}+1} \text { when } \mathrm{r}>\alpha_{n-1}\left(Z_{w}\right) ;
$$

see [15, Corollary 3.17]. (These $j_{\ell}$ have the opposite order of those in [15].) We maintain the convention (A.7). 
It is well-known that the Schubert varieties of $X=\mathcal{S}_{n}$ are indexed by partitions $\lambda=$ $\left(\lambda_{1}, \lambda_{2}, \ldots, \lambda_{n}\right)$ satisfying (A.8) and

$$
\#\left\{i \mid \lambda_{i}>n\right\} \text { is even, }
$$

cf. [1, §9.3]. The corresponding Schubert variety is given by (A.2), with $F^{\bullet}$ an isotropic flag as in Section A.1.

We define $\mathrm{J}(\lambda)$ as in (A.3), with the following modification of the block decomposition. In the block decomposition $\lambda=\hat{\mu}_{p} \cdots \hat{\mu}_{1} \hat{\mu}_{0}$, the integers $n-1, n+1$ are considered 'consecutive' and are placed in the same $\hat{\mu}_{s}$-block; likewise, the integers $n, n+2$ are 'consecutive.' For example, if $n=5$, then $\lambda=(2,3,4,6,10)$ has block decomposition $\hat{\mu}_{1} \hat{\mu}_{0}=(2,3,4,6)(10)$; likewise, $\lambda=(1,2,5,7,8)$ has block decomposition $\hat{\mu}_{1} \hat{\mu}_{0}=(1,2)(5,7,8)$.

As before,

$$
j_{\ell}(\lambda)=\left|\hat{\mu}_{\mathrm{p}} \cdots \hat{\mu}_{\ell}\right| .
$$

Define

$$
\mathrm{a}= \begin{cases}\mathrm{p}-2 & \text { if } \lambda_{1}=1 \text { and } \lambda_{n}-\lambda_{n-1}>1, \\ \mathrm{p}-1 & \text { if } \lambda_{1}=1 \text { and } \lambda_{n}-\lambda_{n-1}=1, \text { or } \lambda_{1}>1 \text { and } \lambda_{n}-\lambda_{n-1}>1, \\ \mathrm{p} & \text { if } \lambda_{1}>1 \text { and } \lambda_{n}-\lambda_{n-1}=1 .\end{cases}
$$

Lemma A.16 ([14]). Given a partition $\lambda$ indexing a Schubert variety (A.2) in $\mathcal{S}_{n}=D_{n} / P_{n}$, the set $\mathrm{J}(\lambda)=\left\{\mathrm{j}_{\mathrm{p}}(\lambda), \ldots, \mathrm{j}_{1}(\lambda)\right\}$ is given by (A.14), and $\mathrm{a}(\lambda)$ is given by (A.15).

Conversely, given a and $\mathrm{J}=\left\{\mathrm{j}_{\mathrm{p}}, \cdots, \mathrm{j}_{1}\right\}$, we construct $\lambda(\mathrm{a}, \mathrm{J})$ as follows. Let $\lambda^{\prime}=$ $\mu_{\mathrm{p}} \cdots \mu_{1} \mu_{0}$ be given by (A.10), with $\ell+m=\mathrm{a}+1+\alpha_{n-1}\left(Z_{w}\right)$. If $\lambda^{\prime}$ satisfies (A.13), then $\lambda=\lambda^{\prime}$. If (A.13) fails for $\lambda^{\prime}$, then we modify the partition as follows: precisely one of $\{n, n+1\}$ belongs to $\lambda^{\prime}$, denote this element by $a^{\prime}$, and the other by $a$. Then $\lambda$ is obtained from $\lambda^{\prime}$ by replacing $a^{\prime}$ with $a$.

As an example, Table 4 lists the partitions and corresponding a : $\mathrm{J}$ and $\mathrm{r}$ values for the Schubert varieties of $\mathcal{S}_{6}=\operatorname{Spin}_{12} \mathbb{C} / P_{6}$.

\section{Appendix B. The exceptional CASES}

Figures 1 and 2 (pages 21 and 22) are respectively the Hasse diagrams $W^{\mathfrak{p}}$ of the Cayley plane $E_{6} / P_{6}$ and Freudenthal variety $E_{7} / P_{7}$. Each node represents a Schubert class $\xi_{w}=$ $\left[Y_{w}\right]$ and is labeled with the corresponding a $(w): \mathrm{J}(w)$ values, which we obtained with the assistance of [11]. The height of the node indicates the dimension of $Y_{w}$; in particular, the lowest node $o \in X$ is at height zero. Two nodes are connected if the Schubert variety

associated with the lower node is a divisor of the Schubert variety associated with the higher node.

\section{REFERENCES}

[1] Sara Billey and V. Lakshmibai. Singular loci of Schubert varieties, volume 182 of Progress in Mathematics. Birkhäuser Boston Inc., Boston, MA, 2000.

[2] Sara Billey and Alexander Postnikov. Smoothness of Schubert varieties via patterns in root subsystems. Adv. in Appl. Math., 34(3):447-466, 2005.

[3] Sara C. Billey and Gregory S. Warrington. Maximal singular loci of Schubert varieties in $\operatorname{SL}(n) / B$. Trans. Amer. Math. Soc., 355(10):3915-3945 (electronic), 2003. 
TABLE 4. Schubert varieties of $\mathcal{S}_{6}$.

\begin{tabular}{|c|c|c||c|c|c|}
\hline$\lambda$ & $\mathrm{a}: \mathrm{J}$ & $\mathrm{r}$ & $\lambda$ & $\mathrm{a}: \mathrm{J}$ & $\mathrm{r}$ \\
\hline \hline$(1,2,3,4,5,6)$ & & & $(1,2,3,4,7,8)$ & $0: 4$ & 0 \\
\hline$(1,2,3,5,7,9)$ & $0: 3,5$ & 1 & $(1,2,4,5,7,10)$ & $0: 2,5$ & 1 \\
\hline$(1,2,3,6,8,9)$ & $0: 3$ & 0 & $(1,3,4,5,7,11)$ & $0: 1,5$ & 1 \\
\hline$(1,2,4,6,8,10)$ & $1: 2,3,5$ & 1 & $(2,3,4,5,7,12)$ & $0: 5$ & 1 \\
\hline$(1,3,4,6,8,11)$ & $1: 1,3,5$ & 1 & $(1,2,5,6,9,10)$ & $1: 2,4$ & 1 \\
\hline$(2,3,4,6,8,12)$ & $1: 3,5$ & 1 & $(1,3,5,6,9,11)$ & $2: 1,2,4,5$ & 2 \\
\hline$(1,2,7,8,9,10)$ & $0: 2$ & 0 & $(2,3,5,6,9,12)$ & $2: 2,4,5$ & 2 \\
\hline$(1,4,5,6,10,11)$ & $1: 1,4$ & 1 & $(1,3,7,8,9,11)$ & $1: 1,2,5$ & 1 \\
\hline$(2,4,5,6,10,12)$ & $2: 1,4,5$ & 2 & $(2,3,7,8,9,12)$ & $1: 2,5$ & 1 \\
\hline$(1,4,7,8,10,11)$ & $2: 1,2,4$ & 1 & $(3,4,5,6,11,12)$ & $1: 4$ & 1 \\
\hline$(2,4,7,8,10,12)$ & $3: 1,2,4,5$ & 2 & $(1,5,7,9,10,11)$ & $1: 1,3$ & 1 \\
\hline$(3,4,7,8,11,12)$ & $2: 2,4$ & 1 & $(2,5,7,9,10,12)$ & $2: 1,3,5$ & 2 \\
\hline$(1,6,8,9,10,11)$ & $0: 1$ & 0 & $(3,5,7,9,11,12)$ & $3: 1,3,4$ & 2 \\
\hline$(2,6,8,9,10,12)$ & $1: 1,5$ & 1 & $(4,5,7,10,11,12)$ & $1: 3$ & 1 \\
\hline$(3,6,8,9,11,12)$ & $2: 1,4$ & 1 & $(4,6,8,10,11,12)$ & $2: 1,3$ & 1 \\
\hline$(5,6,9,10,11,12)$ & $1: 2$ & 1 & $(7,8,9,10,11,12)$ & & \\
\hline
\end{tabular}

[4] Michel Brion and Patrick Polo. Generic singularities of certain Schubert varieties. Math. Z., 231(2):301324, 1999.

[5] Andreas Čap and Jan Slovák. Parabolic geometries. I, volume 154 of Mathematical Surveys and Monographs. American Mathematical Society, Providence, RI, 2009. Background and general theory.

[6] Aurélie Cortez. Singularités génériques et quasi-résolutions des variétés de Schubert pour le groupe linéaire. Adv. Math., 178(2):396-445, 2003.

[7] Vesselin Gasharov. Sufficiency of Lakshmibai-Sandhya singularity conditions for Schubert varieties. Compositio Math., 126(1):47-56, 2001.

[8] Christian Kassel, Alain Lascoux, and Christophe Reutenauer. The singular locus of a Schubert variety. J. Algebra, 269(1):74-108, 2003.

[9] Bertram Kostant. Lie algebra cohomology and the generalized Borel-Weil theorem. Ann. of Math. (2), 74:329-387, 1961.

[10] V. Lakshmibai and B. Sandhya. Criterion for smoothness of Schubert varieties in $\mathrm{Sl}(n) /$ B. Proc. Indian Acad. Sci. Math. Sci., 100(1):45-52, 1990.

[11] LiE. Computer algebra package for semisimple Lie algebra computations, www-math.univpoitiers.fr/ maavl/LiE/.

[12] L. Manivel. Le lieu singulier des variétés de Schubert. Internat. Math. Res. Notices, (16):849-871, 2001.

[13] Nicolas Perrin. The Gorenstein locus of minuscule Schubert varieties. Adv. Math., 220(2):505-522, 2009.

[14] C. Robles. Schur flexibility of cominuscule Schubert varieties. Comm. Anal. Geom. to appear; arXiv:1203.0328.

[15] C. Robles and D. The. Rigid Schubert varieties in compact Hermitian symmetric spaces. Selecta Math. (N.S.), 18(3):717-777, 2012.

[16] Hugh Thomas and Alexander Yong. A combinatorial rule for (co)minuscule Schubert calculus. Adv. Math., 222(2):596-620, 2009.

[17] A. V. Zelevinskiü. Small resolutions of singularities of Schubert varieties. Functional Anal. Appl., $17(2): 142-144,1983$. 
E-mail address: robles@math.tamu.edu

Mathematics Department, Mail-stop 3368, Texas A\&M University, College Station, TX 77843-3368 


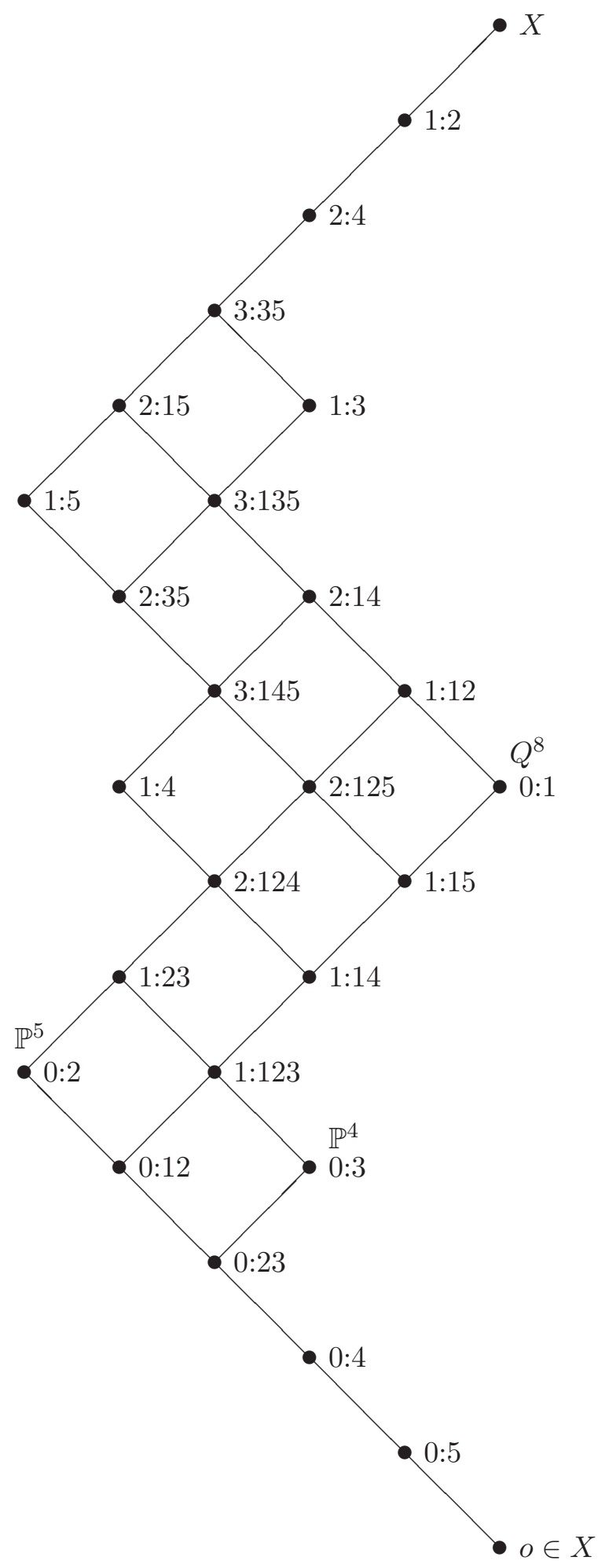

Figure 1. Hasse diagram of $E_{6} / P_{6}$, each node labeled with the a : J values. 


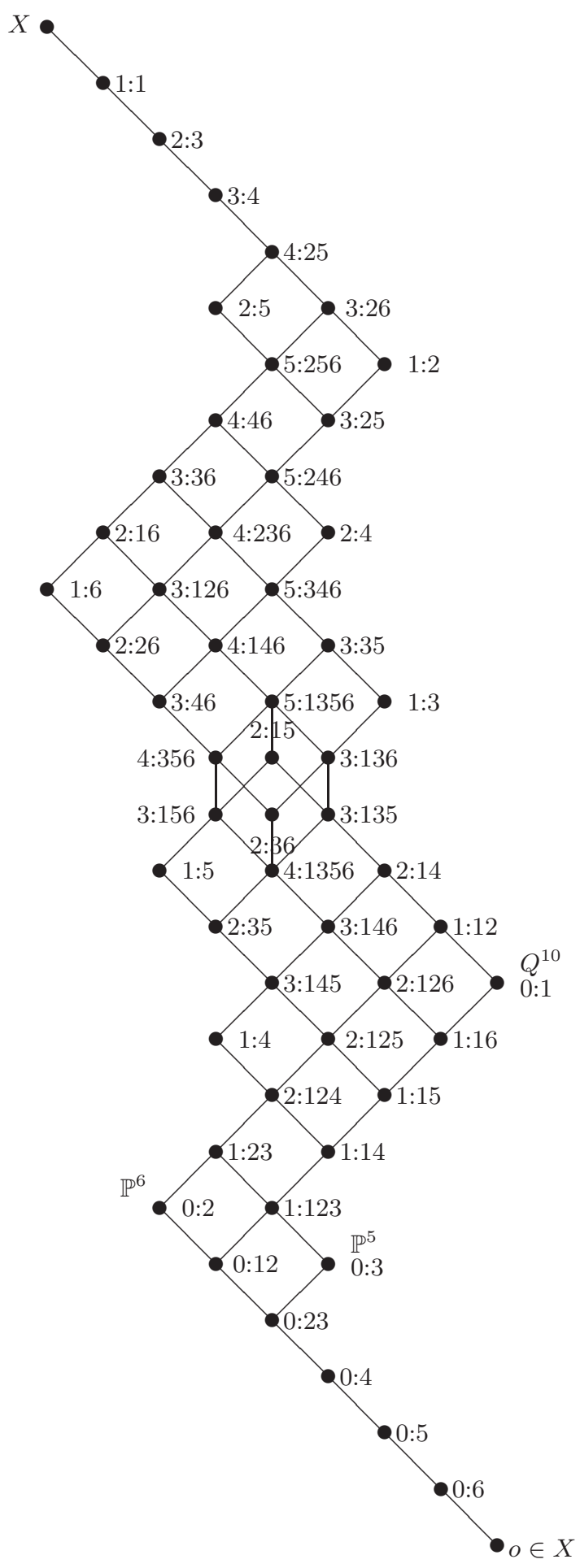

Figure 2. Hasse diagram of $E_{7} / P_{7}$, each node labeled with the a : J values. 